\title{
Homogeneous factorisations of complete graphs with edge-transitive factors
}

\author{
Cai Heng Li · Tian Khoon Lim • Cheryl E. Praeger
}

Received: 20 April 2007 / Accepted: 13 February 2008 / Published online: 26 February 2008

(C) Springer Science+Business Media, LLC 2008

\begin{abstract}
A factorisation of a complete graph $K_{n}$ is a partition of its edges with each part corresponding to a spanning subgraph (not necessarily connected), called a factor. A factorisation is called homogeneous if there are subgroups $M<G \leq S_{n}$ such that $M$ is vertex-transitive and fixes each factor setwise, and $G$ permutes the factors transitively. We classify the homogeneous factorisations of $K_{n}$ for which there are such subgroups $G, M$ with $M$ transitive on the edges of a factor as well as the vertices. We give infinitely many new examples.
\end{abstract}

Keywords Graph factorisation · Edge-transitive graph · Homogeneous factorisation

\section{Introduction}

Let $K_{n}:=(V, E)$ be a complete graph on $n$ vertices, with vertex set $V$ and edge set $E:=\{\{x, y\} \mid x \neq y$ and $x, y \in V\}$. A factorisation of $K_{n}$ is a partition $\mathcal{E}:=$ $\left\{E_{1}, \ldots, E_{k}\right\}$ of $E$ with at least two parts such that each $E_{i}$ is the edge set of a spanning subgraph $\Gamma_{i}=\left(V, E_{i}\right)$, called a factor (that is to say, the set of vertices incident

This paper forms part of an Australian Research Council Discovery Grant project, and was a major part of the PhD project of the second author.

C.H. Li · T.K. Lim · C.E. Praeger $(\bowtie)$

School of Mathematics and Statistics, University of Western Australia, 35 Stirling Highway,

Crawley, WA 6009, Australia

e-mail: praeger@maths.uwa.edu.au

C.H. Li

e-mail: li@maths.uwa.edu.au

Present address:

T.K. Lim

45 Choa Chu Kang Loop \#07-14, 689679, Singapore, Singapore

e-mail: limtk@phillip.com.sg 
with some edge of $E_{i}$ is the whole vertex set $\left.V\right)$. A factorisation $\left(K_{n}, \mathcal{E}\right)$ is said to be ( $G, M)$-homogeneous if $M<G \leq S_{n}, M$ is transitive on $V$ and fixes each factor setwise, while $G$ leaves $\mathcal{E}$ invariant and permutes the factors transitively. Since elements of $G$ induce isomorphisms between the factors, all factors are isomorphic, and indeed 'isomorphic factorisations' of complete graphs have been well studied, see for example $[3,4,13,14]$. Homogeneous factorisations of complete graphs were introduced in [21] (and for graphs in general in [12]). Each vertex-transitive, self-complementary graph $\Gamma$ together with its complement forms a homogeneous factorisation in which the index $k$ is equal to 2 and $\mathcal{E}$ consists of the edge set of $\Gamma$ and the edge set of its complement: we may take $M=\operatorname{Aut}(\Gamma)$ and $G=\langle M, \sigma\rangle$ where $\sigma$ is any permutation that interchanges $\Gamma$ and its complement. Thus all the papers $[1,17,20,26,27,30$, $34]$ give examples of homogeneous factorisations of complete graphs of index 2 .

We give a classification of all $(G, M)$-homogeneous factorisations of $K_{n}$ such that the group $M$ acts edge-transitively on some (and hence all) factors. We call such homogeneous factorisations edge-transitive, and similarly, if each factor is $M$ arc-transitive, then the factorisation is called an arc-transitive homogeneous factorisation. In Section 3 we show that, for each $(G, M)$-homogeneous, edge-transitive factorisation of $K_{n}$, the group $G$ is a 2-homogeneous subgroup of $S_{n}$ and hence is known, see Subsection 2.4. Our classification involves a case-by-case consideration of the families of 2-homogeneous groups, and as the classification of finite 2-homogeneous groups relies on the finite simple group classification, so also does our classification.

Another link with previous work is to the partial classification by Thomas Sibley [29] of decompositions of complete graphs into isomorphic subgraphs (not necessarily spanning subgraphs) preserved by a group acting 2-transitively on the vertex set. His classification is complete in the cases where the automorphism group contains either a simple group or $\operatorname{P\Gamma L}(2,8)$ acting 2-transitively on vertices, but is incomplete in the remaining case where the automorphism group is a subgroup of some affine group $\operatorname{AGL}(d, p)$. The results of this paper provide a classification of those decompositions considered by Sibley that admit 2-transitive affine groups and are homogeneous factorisations.

Our theorem below involves the following notation: the Ree factorisation $\mathcal{E}_{\text {Ree }}(28,3)$ defined in Definition 3.3, the $M$-edge-partition $\mathcal{E}(M)$ defined in Definition 3.2, the cyclotomic factorisations and twisted cycloctomic factorisations defined in Definitions 4.1 and 4.16 respectively, admissible pairs $\left(G_{0}, M_{0}\right)$ defined via Condition 4.12, the graphs $G\left(q^{2}, k\right)$ from Definition 5.4, and the Hamming graph $H(9,2)=\left(\mathbb{Z}_{2}^{9}, E\right)$ where $\{x, y\} \in E$ if and only if $x, y$ differ in exactly one coordinate.

Theorem 1.1 Let $\left(K_{n}, \mathcal{E}\right)$ be a $(G, M)$-homogeneous, edge-transitive factorisation of $K_{n}=(V, E)$ of index $k$ with factors $\Gamma_{i}(1 \leq i \leq k)$. Then $G, M$ may be chosen so that $G$ is 2-transitive on $V$, each factor is $M$-arc-transitive, and one of the following holds.

(1) $(G, M, n, k)=(\operatorname{Ree}(3), \operatorname{PSL}(2,8), 28,3)$, and $\mathcal{E}=\mathcal{E}_{\text {Ree }}(28,3)$.

(2) $G=T \rtimes G_{0}$ is an affine 2-transitive permutation group on $V=V(a, q)$ (where $\left.n=q^{a}\right), M=T \rtimes M_{0}, \mathcal{E}=\mathcal{E}(M)$, and precisely one of the following holds. 
(a) $a=1, \mathcal{E}=\operatorname{Cyc}(q, k)$, and each $\Gamma_{i} \cong \operatorname{GPaley}\left(q, \frac{q-1}{k}\right)$.

(b) $a=1$, and $\left(G_{0}, M_{0}\right)$ is admissible.

(c) $a=2$, and $q, k, M_{0}, \Gamma_{i}$ are as in Tables 2 or 3 .

(d) $a=4, q=3, M_{0}=2^{1+4}, k=5$ and each $\Gamma_{i} \cong H(9,2)$.

Remark 1.2 (a) For Part 2(b), we give in Condition 4.12 an explicit list of parameter conditions that determine whether a pair of subgroups of $\operatorname{A\Gamma L}(1, q)$ is admissible. While there are some examples in Part 2(b) for which $\mathcal{E}(M)=\operatorname{Cyc}(q, k)$, the infinite family of admissible pairs defined explicitly in Proposition 4.18 corresponds to infinitely many new factorisations, the twisted cyclotomic factorisations of Definition 4.16, because the factors of these factorisations, the twisted generalised Paley graphs, are not isomorphic to generalised Paley graphs (unless $(q, k)=(9,2)$, see Proposition 4.18). That is to say, the twisted cyclotomic factorisations are not equivalent to the cyclotomic factorisations of Part 2(a). (We say that two factorisations $\left(K_{n}, \mathcal{E}\right)$ and $\left(K_{n}, \mathcal{E}^{\prime}\right)$ are equivalent if there is a permutation $g \in S_{n}$ such that $E^{g} \in \mathcal{E}^{\prime}$ for each $E \in \mathcal{E}$.)

(b) In some of the examples in Theorem 1.1 the factors are not connected. This is true in particular for a sub-family of the cycloctomic factorisations, see Lemma 4.3.

(c) More details about the examples in Part 2(c) are given in Remark 5.7. Moreover, Lemma 5.6 determines precisely which of these factorisations are equivalent to a factorisation in Part 2(a) or 2(b). Even though the $\Gamma_{i}$ are sometimes generalised Paley graphs or twisted generalised Paley graphs, in most cases the factorisation is new.

(d) The exceptional almost simple example in Part 1 is associated with Ree(3), the only almost simple 2-transitive group whose socle is not 2-transitive.

(e) The exceptional example in Part 2(d) is associated with the exceptional affine 2-transitive group $G$ with $G_{0} \leq 2^{1+4} \cdot S_{5}$, and $M_{0}=2^{1+4}$; it is explored in detail in [23] and [24].

After presenting some preliminary results in Section 2, we give the proof of Theorem 1.1 in the following three sections.

\section{Preliminaries}

\subsection{Graphs}

All graphs considered in this paper are finite, undirected and without loops or multiple edges. Thus a graph $\Gamma=(V \Gamma, E \Gamma)$ consists of a vertex set $V \Gamma$ and a set $E \Gamma$ of unordered pairs of vertices, called edges. If $\{\alpha, \beta\} \in E \Gamma$, then $\alpha$ and $\beta$ are said to be adjacent and the ordered pairs $(\alpha, \beta)$ and $(\beta, \alpha)$ are called arcs. The set of arcs of $\Gamma$ is denoted $A \Gamma$. An automorphism of $\Gamma$ is a permutation of $V \Gamma$ that leaves $E \Gamma$ invariant. The set of all automorphisms forms a subgroup $\operatorname{Aut}(\Gamma)$ of $\operatorname{Sym}(V \Gamma)$ (the symmetric group on $V \Gamma$ ), and is called the automorphism group of $\Gamma$, and for a subgroup $G \leq \operatorname{Aut}(\Gamma), \Gamma$ is $G$-vertex-transitive, $G$-edge-transitive or $G$-arc-transitive if $G$ acts transitively on the vertices, edges or arcs of $\Gamma$ respectively. An arc-transitive 
graph is also edge-transitive but the converse is not true in general. Arc-transitivity is characterised in the following lemma (see [2]). For $v \in V \Gamma, \Gamma(v)$ denotes the set of vertices adjacent to $v$.

Lemma 2.1 A connected graph $\Gamma$ is $G$-arc-transitive if and only if $G$ is transitive on $V \Gamma$ and for any $v \in V \Gamma, G_{v}$ is transitive on $\Gamma(v)$.

\subsection{Cayley graphs}

We will often encounter a special kind of vertex-transitive graph called a Cayley graph, defined as follows.

Definition 2.2 For a group $G$ and a nonempty subset $S$ of $G$ such that $1_{G} \notin S$ and $S=S^{-1}=\left\{s^{-1} \mid s \in S\right\}$, the Cayley graph $\Gamma=\operatorname{Cay}(G, S)$ of $G$ relative to $S$ is defined as the graph with vertex set $V \Gamma=G$ and edge set $E \Gamma$ such that

$$
\{x, y\} \in E \Gamma \Longleftrightarrow y x^{-1} \in S .
$$

A Cayley graph $\operatorname{Cay}(G, S)$ is connected if and only if $\langle S\rangle=G$ (see [7, p. 241]).

A permutation group $G$ on $V$ is semiregular if the only element fixing a point in $V$ is the identity element of $G$. A group $G$ is regular on $V$ if it is both semiregular and transitive, and such groups characterise Cayley graphs as follows.

Lemma 2.3 [2, Lemma 16.3]. A graph $\Gamma$ is isomorphic to a Cayley graph for some group if and only if some subgroup of $\operatorname{Aut}(\Gamma)$ is regular on vertices.

\subsection{Permutation groups}

A partition of a set $V$ is a family $\mathcal{B}$ of non-empty subsets of $V$ such that $\cup_{B \in \mathcal{B}} B=V$ and $B \cap B^{\prime}=\varnothing$ for distinct $B, B^{\prime} \in \mathcal{B}$. Let $G$ be a group acting on a finite set $V$. A nonempty subset $B \subseteq V$ is called a block for $G$ if for every $g \in G$, either $B \cap B^{g}=\varnothing$ or $B=B^{g}$. A block $B$ is said to be trivial if $|B|=1$ or $B=V$. Otherwise, $B$ is nontrivial. A partition $\mathcal{B}$ of $V$ is called $G$-invariant if $B^{g} \in \mathcal{B}$ for any $B \in \mathcal{B}$ and $g \in G$. It is easy to see that the parts of a $G$-invariant partition $\mathcal{B}$ are blocks for $G$, and that $G$ permutes the elements of $\mathcal{B}$ blockwise inducing a natural, possibly unfaithful, action on $\mathcal{B}$. The group $G$ is primitive on $V$ if $G$ is transitive and the only blocks for $G$ are the trivial ones. If $G$ is transitive but not primitive on $V$, then $G$ is said to be imprimitive. The lemma below shows that partitions invariant under a transitive permutation group often arise as sets of orbits of normal subgroups. For a group $G$ acting on a set $V$, and a point $v \in V$, we denote the $G$-orbit containing $v$ by $v^{G}=\left\{v^{g} \mid g \in G\right\}$.

Lemma 2.4 Let $G \leq \operatorname{Sym}(V)$, let $M$ be a normal subgroup of $G$ and let $\mathcal{B}$ be the set of $M$-orbits in $V$. Then $\left(v^{M}\right)^{g}=\left(v^{g}\right)^{M}$ for each $v^{M} \in \mathcal{B}$ and $g \in G$. Moreover $\mathcal{B}$ is $a G$-invariant partition of $V$, and if $G$ is transitive on $V$ then $G$ acts transitively on $\mathcal{B}$, and all $M$-orbits in $V$ have the same length. 
Proof Let $v^{M} \in \mathcal{B}$ and $g \in G$. Then $u \in\left(v^{M}\right)^{g}$ if and only if $u=v^{m g}$ for some $m \in$ $M$. Now $v^{m g}=\left(v^{g}\right)^{g^{-1} m g}$, and $g^{-1} m g$ runs through all the elements of $M$ as $m$ runs through $M$. Thus $\left(v^{M}\right)^{g}=\left(v^{g}\right)^{M} \in \mathcal{B}$, and hence $\mathcal{B}$ is a $G$-invariant partition of $V$. Now assume that $G$ is transitive on $V$. Then for $v^{M}, u^{M} \in \mathcal{B}$, there exists $g \in G$ such that $v^{g}=u$ and hence such that $\left(v^{M}\right)^{g}=\left(v^{g}\right)^{M}=u^{M}$ and $\left|v^{M}\right|=\left|\left(v^{M}\right)^{g}\right|=\left|u^{M}\right|$. This establishes the remaining assertions.

Groups $G, H$ acting on sets $V, U$ respectively, are said to be permutationally isomorphic if there exist a group isomorphism $\theta: G \longrightarrow H$ and a bijection $\xi: V \longrightarrow U$ such that $(\xi(v))^{\theta(g)}=\xi\left(v^{g}\right)$ for all $g \in G$ and $v \in V$.

\subsection{Finite 2-homogeneous groups}

Let $G \leq \operatorname{Sym}(V)$ with $V$ finite. Then $G$ is 2-homogeneous if it is transitive on the set of 2-element subsets of $V$, and $G$ is 2-transitive if it is transitive on the set of ordered pairs of distinct elements of $V$. Such groups are known to be almost simple or affine, defined as follows.

The group $G$ is almost simple if it has a unique minimal normal subgroup $\operatorname{Soc}(G)$ which is a nonabelian simple group. Equivalently, $G$ is almost simple if $T \leq G \leq \operatorname{Aut}(T)$ for some nonabelian simple group $T$ (since an almost simple group $G$ can be embedded as a subgroup of $\operatorname{Aut}(\operatorname{Soc}(G))$ containing the group of inner automorphisms).

The group $G$ is affine if it has an elementary abelian regular normal subgroup $T \cong$ $\mathbb{Z}_{p}^{R}$, for some prime $p$. In this case we may identify $V$ with an $R$-dimensional vector space over a field of order $p$ such that $G$ becomes a group of affine transformations, namely $G=T \rtimes G_{0} \leq \operatorname{AGL}(R, p)$ with $T$ the group of translations $\left(t_{x}: v \longrightarrow v+x\right.$, for all $x, v \in V)$ and $G_{0} \leq \operatorname{GL}(R, p)$, the stabiliser of the zero-vector $\mathbf{0} \in V$. The finite 2-homogeneous but not 2-transitive groups were characterised by Kantor [18] (or see [16, pp. 368-369]) as 1-dimensional affine groups, while the finite affine 2transitive groups were classified by Hering and are listed in [19] and [22, Appendix], and the finite almost simple 2-transitive groups are described in [9, Section 7.7]. These classifications rely on the finite simple group classification, and we state the parts of the classification that we will use in this paper below.

Theorem 2.5 Let $G$ be a finite 2-homogeneous permutation group on a set $V$ with $|V|=n$. Then $G$ has a unique minimal normal subgroup $T$ and one of the following holds:

(1) $T$ is nonabelian simple and either $T$ is 2-transitive, or $T=\operatorname{PSL}(2,8), n=28$ and $G=\mathrm{P} \Gamma \mathrm{L}(2,8)$ is 2-transitive.

(2) $T=\mathbb{Z}_{p}^{R}$ for some prime $p$ and integer $R \geq 1$, and $G=T \rtimes G_{0}$ is affine, with $G_{0} \leq \Gamma \mathrm{L}(a, q)$ where $q^{a}=p^{R}$. Moreover, either $G_{0}$ has 2-orbits $X$ and $-X$ in $V \backslash\{\mathbf{0}\}$, or $G$ is 2-transitive and $G_{0}$ is one of the following (note that the symbol "o" denotes a central product):

(a) $a=1$ and $G_{0} \leq \Gamma \mathrm{L}(1, q)$,

(b) $a \geq 2$ and $\Gamma \mathrm{L}(a, q) \geq G_{0} \unrhd \operatorname{SL}(a, q)$,

(c) $a=2 l \geq 4$ and $\mathbb{Z}_{q-1} \circ \Gamma \operatorname{Sp}(a, q) \geq G_{0} \unrhd \operatorname{Sp}(a, q)$, 
(d) $a=6$, $q$ even and $\mathbb{Z}_{q-1} \times \operatorname{Aut}\left(G_{2}(q)\right) \geq G_{0} \unrhd G_{2}(q)^{\prime}$,

(e) $a=4, q=2$ and $G_{0} \cong A_{6}$ or $A_{7}$,

(f) $a=6, q=3$ and $G_{0}=\operatorname{SL}(2,13)$,

(g) $a=2, q=p=5,7,11$ or 23 and $G_{0} \unrhd \mathrm{SL}(2,3)$, or $a=2, q=9,11,19,29$ or 59 and $G_{0} \unrhd \operatorname{SL}(2,5)$,

(h) $a=4, q=3$ and $G_{0}$ has a normal extraspecial subgroup $\mathbf{E}$ of order $2^{5}$, and $G_{0} / \mathbf{E}$ is isomorphic to a subgroup of $S_{5}$.

Remark 2.6 In Theorem 2.5(2), $G$ preserves on $V$ the structure of an $a$-dimensional vector space over the finite field $\mathbb{F}_{q}$. Thus $G_{0} \leq \Gamma \mathrm{L}(a, q)$ and $V=\mathbb{F}_{q}^{a}=V(a, q)$ where $q^{a}=p^{R}$ with $a \geq 1$. Also in cases (a)-(h), $G_{0}$ is transitive on the set of nonzero vectors in $V$, denoted as $V^{*}$.

\subsection{Orbitals}

Now let $G \leq \operatorname{Sym}(V)$ be transitive on $V$. Then $G$ acts faithfully on the set $V \times V$ via the action $\left(v_{1}, v_{2}\right)^{g}=\left(v_{1}^{g}, v_{2}^{g}\right)$ where $v_{1}, v_{2} \in V$ and $g \in G$. The orbits of $G$ on $V \times V$ are known as the orbitals of $G$ in $V$ (or simply the $G$-orbitals). In particular $\{(v, v) \mid v \in V\}$ is a $G$-orbital, called the trivial orbital, and all others are called nontrivial. Furthermore for each $G$-orbital $\mathcal{O}=(u, v)^{G}$, the $G$-orbital $\mathcal{O}^{*}=(v, u)^{G}$ is called the paired orbital of $\mathcal{O}$; if $\mathcal{O}=\mathcal{O}^{*}$ then $\mathcal{O}$ is said to be self-paired.

Lemma 2.7 Let $G \leq \operatorname{Sym}(V)$ with a transitive normal subgroup $M$. Then $G$ leaves invariant the set of nontrivial $M$-orbitals, and the set of self-paired, non-trivial $M$ orbitals.

Proof Let $\mathcal{O}=(u, v)^{M}$ be a nontrivial $M$-orbital and $g \in G$. Since $G$ acts faithfully on $V \times V$, it follows from Lemma 2.4 that $\mathcal{O}^{g}=\left((u, v)^{g}\right)^{M}=\left(u^{g}, v^{g}\right)^{M}$, which is also a nontrivial $M$-orbital. Moreover, if $\mathcal{O}^{*}=\mathcal{O}$ then $\left(\mathcal{O}^{g}\right)^{*}=\left(v^{g}, u^{g}\right)^{M}=$ $\left((v, u)^{M}\right)^{g}=\left((u, v)^{M}\right)^{g}=\mathcal{O}^{g}$.

For a transitive subgroup $G \leq \operatorname{Sym}(V)$, to each non-trivial $G$-orbital $\mathcal{O}$ we associate a graph $\Gamma(\mathcal{O})=(V, E(\mathcal{O}))$ where $E(\mathcal{O})=\left\{\{x, y\} \mid(x, y) \in \mathcal{O} \cup \mathcal{O}^{*}\right\}$. Then $\Gamma(\mathcal{O})=\Gamma\left(\mathcal{O}^{*}\right), \Gamma(\mathcal{O})$ has arc set $\mathcal{O} \cup \mathcal{O}^{*}$, is $G$-edge-transitive for any $\mathcal{O}$, and is $G$ arc-transitive if and only if $\mathcal{O}=\mathcal{O}^{*}$. Moreover the converse holds (see for example [28, Theorem 2.1 (b)] or [32, 7.53 on p. 59]).

Lemma 2.8 For a transitive subgroup $G \leq \operatorname{Sym}(V)$ and graph $\Gamma=(V, E)$ with $E \neq \emptyset$,

(1) $\Gamma$ is $G$-arc-transitive if and only if $E=E(\mathcal{O})$ for some non-trivial self-paired $G$-orbital $\mathcal{O}$, and

(2) $\Gamma$ is $G$-edge-transitive, but not $G$-arc-transitive, if and only if $E=E(\mathcal{O})$ for some non-trivial $G$-orbital $\mathcal{O}$ such that $\mathcal{O} \neq \mathcal{O}^{*}$.

For $G, \mathcal{O}$ as above, and for a point $v \in V$, the $\operatorname{set} \mathcal{O}(v):=\{u \mid(v, u) \in \mathcal{O}\}$ is a $G_{v^{-}}$ orbit in $V \backslash\{v\}$, and each such $G_{v}$-orbit arises as $\mathcal{O}(v)$ for some non-trivial $G$-orbital 
$\mathcal{O}$ (see [9, Section 3.2]). Moreover, $\mathcal{O}(v) \cup \mathcal{O}^{*}(v)$ is the set $\Gamma(v)$ of vertices adjacent to $v$ in the graph $\Gamma=\Gamma(\mathcal{O})$.

\subsection{Affine Cayley graphs}

Most of our effort is directed towards considering affine subgroups $G \leq \operatorname{Sym}(V)$ with regular minimal normal subgroup $T=\mathbb{Z}_{p}^{R}$, and consequently (see Lemma 2.3) the graphs $\Gamma(\mathcal{O})$ are Cayley graphs for $T$. We interpret $V$ as a finite vector space over some extension field $\mathbb{F}_{q}$ of $\mathbb{F}_{p}$ and $T$ as the translation group $T=\left\{t_{x} \mid x \in V\right\}$ (written additively) of $V$, where $t_{x}: v \longrightarrow v+x$. Thus $G=T \rtimes G_{0}$ with $G_{0} \leq \Gamma \mathrm{L}(a, q)$, the stabiliser of the zero vector $\mathbf{0}$. If $G \leq \operatorname{Aut}(\Gamma)$ with $\Gamma=(V, E)$, then $\Gamma=\operatorname{Cay}(T, S)$ for some $G_{0}$-invariant $S \subset T$ with $S=-S$. We bring together the orbital description of edge-transitive Cayley graphs and their definition as Cayley graphs.

Lemma 2.9 Let $G=T \rtimes G_{0}$ be an affine transitive permutation group on the vector space $V=V(a, q)$ where $T=\mathbb{Z}_{p}^{R}, G_{0} \leq \Gamma \mathrm{L}(a, q)$ and $q^{a}=p^{R}$. Let $\Gamma=\operatorname{Cay}(V, S)$ be a Cayley graph on $V$ (with $S=-S$ ) and suppose that $G \leq \operatorname{Aut}(\Gamma)$. Then the following hold.

(1) $\Gamma$ is $G$-arc-transitive if and only if $S=\mathcal{O}(\mathbf{0})$ for a non-trivial self-paired $G$ orbital $\mathcal{O}$.

(2) $\Gamma$ is $G$-edge-transitive but not $G$-arc-transitive if and only if $S=\mathcal{O}(\mathbf{0}) \cup \mathcal{O}^{*}(\mathbf{0})$, for a non-trivial $G$-orbital $\mathcal{O}$ with $\mathcal{O} \neq \mathcal{O}^{*}$. Moreover $\mathcal{O}^{*}(\mathbf{0})=-\mathcal{O}(\mathbf{0})$, and in this case $q$ is odd.

Proof The 'connecting set' $S$ of the Cayley graph $\Gamma=\operatorname{Cay}(V, S)$ is precisely the set of vertices adjacent to the zero-vector 0. Part 1 then follows from Lemma 2.1 and the remarks following. For part 2, recall from Lemma 2.8(2) that $\Gamma=\operatorname{Cay}(V, S)$ is $G$-edge-transitive but not $G$-arc-transitive if and only if $E \Gamma=E(\mathcal{O})$ for some nontrivial $G$-orbital $\mathcal{O}$ with $\mathcal{O} \neq \mathcal{O}^{*}$, that is to say, if and only if $S=\mathcal{O}(\mathbf{0}) \cup \mathcal{O}^{*}(\mathbf{0})$. Let $s \in \mathcal{O}(\mathbf{0})$ and consider the action of the translation $t_{-s} \in T$. Now $(\mathbf{0}, s)^{t_{-s}}=$ $(-s, \mathbf{0}) \in \mathcal{O}$ and so $(\mathbf{0},-s) \in \mathcal{O}^{*}$. Thus $\mathcal{O}^{*}(\mathbf{0})=(-s)^{G_{0}}=-\left(s^{G_{0}}\right)=-\mathcal{O}(\mathbf{0})$. Since in this case $\mathcal{O}(\mathbf{0}) \neq \mathcal{O}^{*}(\mathbf{0})$ it follows that $q$ is odd.

\section{Reduction to the affine case}

Throughout the rest of the paper we assume that $\left(K_{n}, \mathcal{E}\right)$ is a $(G, M)$-homogeneous edge-transitive factorisation. We write $K_{n}=(V, E)$, and $\mathcal{E}=\left\{E_{1}, \ldots, E_{k}\right\}$ with corresponding factors $\Gamma_{i}=\left(V, E_{i}\right)$, for $1 \leq i \leq k$.

Lemma 3.1 The group $G$ is 2-homogeneous on $V$.

Proof Let $\{x, y\}$ and $\{u, v\}$ be two-element subsets of $V$. Since $G$ is transitive on $\mathcal{E}$, some element of $G$ maps $\{x, y\}$ to a pair $\left\{x^{\prime}, y^{\prime}\right\}$ lying in the same part of $\mathcal{E}$ as $\{u, v\}$, say $E_{i}$. Then since $\Gamma_{i}$ is $M$-edge-transitive, some element of $M$ maps $\left\{x^{\prime}, y^{\prime}\right\}$ to $\{u, v\}$. Thus $G$ is 2-homogeneous. 
If $\left(K_{n}, \mathcal{E}\right)$ is $(G, M)$-homogeneous arc-transitive then $M$ has exactly $k$ nontrivial orbitals, each self-paired, and they may be labeled so that $E_{i}=E\left(\mathcal{O}_{i}\right)$ and $\Gamma_{i}=\Gamma\left(\mathcal{O}_{i}\right)$ for $i=1, \ldots, k$. It is convenient to have a standard notation for the corresponding edge-partition.

Definition 3.2 Suppose that a transitive subgroup $M \leq \operatorname{Sym}(V)$ has $k$ non-trivial orbitals $\mathcal{O}_{1}, \ldots, \mathcal{O}_{k}$ and each is self-paired. The $M$-edge-partition is the partition $\mathcal{E}(M):=\left\{E\left(\mathcal{O}_{i}\right) \mid 1 \leq i \leq k\right\}$.

Before continuing with the general discussion we make some comments about almost simple 2-transitive groups $G$. The simple normal subgroup $T$ of $G$ is 2transitive except when $G=\operatorname{Ree}(3)$ is the smallest Ree group acting on 28 points and $T=G^{\prime} \cong \operatorname{PSL}(2,8)$ (see [6] or [9, p. 245-253]). This exceptional group gives rise to a homogeneous arc-transitive factorisation of index 3 .

Definition 3.3 Let $G=\operatorname{Ree}(3)$ acting 2-transitively on a set $V$ of size 28 and let $M=$ $G^{\prime} \cong \operatorname{PSL}(2,8)$. There are exactly three non-trivial $M$-orbitals $\mathcal{O}_{1}, \mathcal{O}_{2}, \mathcal{O}_{3}$, each selfpaired, and these are permuted transitively by $G$. Let $\Gamma_{i}:=\Gamma\left(\mathcal{O}_{i}\right)=\left(V, E\left(\mathcal{O}_{i}\right)\right)$ for $i=1,2,3$ (as defined before Lemma 2.8), and let $\mathcal{E}_{\text {Ree }}(28,3)=\mathcal{E}(M)$. Then $\left(K_{28}, \mathcal{E}_{\text {Ree }}(28,3)\right)$ is a $(G, M)$-homogeneous arc-transitive factorisation.

We note that each of the factors $\Gamma\left(\mathcal{O}_{i}\right)$ is $M$-arc-transitive of valency 9. Moreover, using MaGMA [8] it is simple to check that $\Gamma\left(\mathcal{O}_{i}\right)$ has automorphism group $M$ and is not a Cayley graph. We prove below that this is the only homogeneous edge-transitive factorisation of a complete graph with the group $G$ almost simple, and hence also the only example for which the factors are not Cayley graphs. We also prove the first assertion of Theorem 1.1.

Proposition 3.4 Replacing $(G, M)$ if necessary by slightly larger groups, we may assume that $G$ is 2-transitive on $V, M \triangleleft G$, and each $\Gamma_{i}$ is $M$-arc-transitive. Moreover, either $G, M, n, \mathcal{E}$ are as in Definition 3.3, or

(1) $G=T \rtimes G_{0}$ and $M=T \rtimes M_{0}$ are affine with $T=\mathbb{Z}_{p}^{R}, M_{0} \triangleleft G_{0} \leq \Gamma \mathrm{L}(a, q)$, $\phi \in M_{0}$ (where $\phi: v \longrightarrow-v$, for all $\left.v \in V\right), n=q^{a}$, and $V=V(a, q)$, an a-dimensional vector space over $\mathbb{F}_{q}$;

(2) $\mathcal{E}=\mathcal{E}(M)$ as defined in Definition 3.2.

Proof Since $M$ fixes each of the $E_{i}$ setwise, $M$ is contained in the kernel of the $G$-action on $\mathcal{E}$. Replacing $M$ if necessary by this kernel we may assume from now on that $M \triangleleft G$. Since $k \geq 2, M$ is not transitive on the $\operatorname{arcs}$ of $K_{n}$, and so $M$ is not 2-transitive. By Lemma 3.1 the group $G$ is 2-homogeneous on $V$, and hence by Theorem 2.5, either $G$ is affine or $G$ is almost simple and 2-transitive. In either case $G$ has a unique minimal normal subgroup $T$, and so we must have $T \leq M$. In particular $T$ is not 2-transitive.

In the almost simple case, as discussed above, the simple normal subgroup $T$ of $G$ is 2-transitive, except when $G=\operatorname{Ree}(3)$ on 28 points and $T=G^{\prime} \cong \operatorname{PSL}(2,8)$. Thus in this case $G, M, n$ are as in Definition 3.3. Also as the group PSL(2,8) has three 
non-trivial orbitals, each self-paired, and permuted transitively by $G$, it follows from Lemma 2.8 that $k=3, \mathcal{E}$ is as in Definition 3.3, and each $\Gamma_{i}$ is $M$-arc-transitive. Thus the result is proved in this case.

We assume from now on that $G$ is affine, so $G=T \rtimes G_{0}$ with $T=\mathbb{Z}_{p}^{R}, G_{0} \leq$ $\Gamma \mathrm{L}(a, q)$, where $n=q^{a}=p^{R}$, and $V$ is identified with an $a$-dimensional vector space $V(a, q)$ over $\mathbb{F}_{q}$. Since $T \leq M \triangleleft G$, we have $M=T \rtimes M_{0}$ and $M_{0} \triangleleft G_{0}$. As discussed in Subsection 2.2, each $\Gamma_{i}=\operatorname{Cay}\left(T, S_{i}\right)$ for some $M_{0}$-invariant subset $S_{i} \subset T$ with $S_{i}=-S_{i}$. By assumption $\Gamma_{i}$ is $M$-edge-transitive and so, by Lemnma 2.9, there is a non-trivial $M$-orbital $\mathcal{O}_{i}$ such that $S_{i}=\mathcal{O}_{i}(\mathbf{0})$ if $\Gamma_{i}$ is $M$-arctransitive, or $S_{i}=\mathcal{O}_{i}(\mathbf{0}) \cup \mathcal{O}_{i}^{*}(\mathbf{0})$ with $\mathcal{O}_{i} \neq \mathcal{O}_{i}^{*}$ if $\Gamma_{i}$ is not $M$-arc-transitive. In the latter case $\mathcal{O}_{i}^{*}(\mathbf{0})=-\mathcal{O}_{i}(\mathbf{0})$, and in either case $E_{i}=E\left(\mathcal{O}_{i}\right)$ and $\Gamma_{i}=\Gamma\left(\mathcal{O}_{i}\right)$. Now the transformation $\phi \in \operatorname{GL}(a, q)$ fixes $\mathbf{0}$ and interchanges $\mathcal{O}_{i}(\mathbf{0})$ and $-\mathcal{O}_{i}(\mathbf{0})=\mathcal{O}_{i}^{*}(\mathbf{0})$. Hence $\phi$ fixes $E\left(\mathcal{O}_{i}\right)$ for each $i$ and also $\phi$ centralises $G$. Thus the group $\langle G, \phi\rangle$ is 2-transitive on $V$ and leaves $\mathcal{E}$ invariant, and its subgroup $\langle M, \phi\rangle$ is normal and fixes each $E_{i}$ setwise. Replacing $G, M$ by these groups we may therefore assume that $\phi \in M_{0}$. This implies that $M_{0}$ is transitive on $S_{i}$ for each $i$ and hence, by Lemma 2.1, each $\Gamma_{i}$ is $M$-arc-transitive and $G$ is 2-transitive. Also each of the $\mathcal{O}_{i}(1 \leq i \leq k)$ is self-paired and these are all of the non-trivial $M$-orbitals, so $\mathcal{E}=\mathcal{E}(M)$.

In the light of Proposition 3.4, we assume from now on that $G$ is an affine 2transitive permutation group on $V$ that contains the map $\phi: v \longrightarrow-v$ (for $v \in V$ ). Our broad strategy is to consider each of the possibilities for $G$, as given in Theorem 2.5(2), examine each of its normal subgroups $M$ that is not 2-homogeneous and contains $\phi$, and determine the structure of the $M$-arc-transitive factors. Thus in the remainder of the paper we assume that $V=V(a, q)$ where $|V|=q^{a}=p^{R}$ with $p$ prime, and $G, M$ satisfy the following condition.

Condition 3.5 $G=T \rtimes G_{0}, M=T \rtimes M_{0}$ with $T=\mathbb{Z}_{p}^{R}, M_{0} \triangleleft G_{0} \leq \Gamma \mathrm{L}(a, q)$ and $\phi \in M_{0}$.

Also $M$ has $k$ non-trivial orbitals $\mathcal{O}_{1}, \ldots, \mathcal{O}_{k}$, each self-paired, and such that $E_{i}=E\left(\mathcal{O}_{i}\right)$, and $\Gamma_{i}=\Gamma\left(\mathcal{O}_{i}\right)=\operatorname{Cay}\left(V, S_{i}\right)$ with $S_{i}=\mathcal{O}_{i}(\mathbf{0})$, for $i=1, \ldots, k$. Note that we identify the vertex set $V$ with $T$, and without loss of generality we will assume that $1 \in S_{1}$. Thus $\left(K_{n}, \mathcal{E}\right)=\left(K_{p^{R}}, \mathcal{E}(M)\right)$, where $\mathcal{E}(M)=\left\{E\left(\mathcal{O}_{1}\right), \ldots E\left(\mathcal{O}_{k}\right)\right\}$ as in Definition 3.2, and this is a $(G, M)$-homogeneous factorisation if and only if $G$ acts transitively on $\left\{\mathcal{O}_{1}, \ldots, \mathcal{O}_{k}\right\}$ (with the action described in Lemma 2.7), or equivalently $G_{0}$ acts transitively on $\left\{S_{1}, \ldots, S_{k}\right\}$.

\section{The one-dimensional affine case}

This is case (a) of Theorem 2.5(2). The group $G$ is as in Condition 3.5 with $a=1$. In this case we identify $V$ with the finite field $\mathbb{F}_{q}$ of order $q=p^{R}$. We introduce generators for $\Gamma \mathrm{L}(1, q)$ as follows. Choose $\omega$ to be a primitive element of $\mathbb{F}_{q}$ and denote by $\widehat{\omega}$ the corresponding scalar multiplication $x \longrightarrow x \omega$ (for $x \in V$ ). Also let $\alpha$ denote the Frobenius automorphism of $\mathbb{F}_{q}$, that is, $\alpha: x \longrightarrow x^{p}$. Then $\widehat{\omega}$ generates the multiplicative group $\operatorname{GL}(1, q), \Gamma \mathrm{L}\left(1, p^{R}\right)=\langle\widehat{\omega}, \alpha\rangle$ and $\mathrm{A} \Gamma \mathrm{L}(1, q)=T \rtimes\langle\widehat{\omega}, \alpha\rangle$. 
Although it is necessary to distinguish elements of $\operatorname{GL}(1, q)$ from elements of $V$, it is helpful to have similar notation for certain subsets of $V$. For $i \geq 1$ and $i \mid(q-1)$, we shall use $\left\langle\omega^{i}\right\rangle$ to denote the subset $\left\{1, \omega^{i}, \omega^{2 i}, \ldots, \omega^{((q-1) / i)-i}\right\}$ of $V$. Also, for $1,-1 \in \mathbb{F}_{q}$, we will use $\widehat{1}$ and $\widehat{-1}$ to denote the corresponding scalar multiplications in $\operatorname{GL}(1, q)$.

\subsection{Generalised Paley graphs and cyclotomic factorisations}

First we give a family of examples generalising the homogeneous factorisation consisting of the edge sets of a Paley graph and its complement.

Definition 4.1 (Generalised Paley graph and cyclotomic partition) Let $k$ be a divisor of $q-1$ such that $k \geq 2$ and either $q$ or $\frac{q-1}{k}$ is even. Then the graph $\operatorname{GPaley}\left(q, \frac{q-1}{k}\right)=\operatorname{Cay}\left(V,\left\langle\omega^{k}\right\rangle\right)$ is called a generalised Paley graph on $V$. The corresponding cyclotomic partition of $K_{q}$ is the partition $\operatorname{Cyc}(q, k)=\left\{E_{1}, \ldots, E_{k}\right\}$ where $E_{i}=\left\{\{u, v\} \mid v-u \in \omega^{i-1}\left\langle\omega^{k}\right\rangle\right\}$ for $1 \leq i \leq k$.

Note that the conditions on $k$ imply that $\left\langle\omega^{k}\right\rangle=-\left\langle\omega^{k}\right\rangle$ and so the graph $\operatorname{GPaley}\left(q, \frac{q-1}{k}\right)$ is well defined as an undirected Cayley graph. If $k=2$ then $\operatorname{GPaley}\left(q, \frac{q-1}{2}\right)$ is the Paley graph which is arc-transitive and self-complementary, with automorphism group $T \rtimes\left\langle\widehat{\omega}^{2}, \alpha\right\rangle$ (see for example [27]). In Proposition 4.2 we prove that $\left(K_{q}, \operatorname{Cyc}(q, k)\right)$ is a homogeneous factorisation, called a cyclotomic factorisation. These factorisations are closely related to cyclotomic association schemes, see for example [5, Section 2.10A].

Proposition 4.2 Let $k$ be a divisor of $q-1$ such that $k \geq 2$ and either $q$ or $\frac{q-1}{k}$ is even. Let $G=T \rtimes\langle\widehat{\omega}\rangle$ and $M=T \rtimes\left\langle\widehat{\omega}^{k}\right\rangle$.

(1) Then $\operatorname{GPaley}\left(q, \frac{q-1}{k}\right)$ is an undirected, $M$-arc-transitive graph of valency $\frac{q-1}{k}$.

(2) The pair $\left(K_{q}, \operatorname{Cyc}(q, k)\right)$ is a $(G, M)$-homogeneous arc-transitive factorisation of index $k$, and each factor is isomorphic to $\operatorname{GPaley}\left(q, \frac{q-1}{k}\right)$.

Proof Part 1. The 'connecting set' $\left\langle\omega^{k}\right\rangle$ is an orbit for $M_{0}=\left\langle\widehat{\omega}^{k}\right\rangle$ of size $\frac{q-1}{k}$, and hence is equal to $\mathcal{O}(\mathbf{0})$ for a non-trivial $M$-orbital $\mathcal{O}$. As discussed above $\mathcal{O}(\mathbf{0})=$ $-\mathcal{O}(\mathbf{0})$ and so, by Lemma 2.9, $\operatorname{GPaley}\left(q, \frac{q-1}{k}\right)$ is $M$-arc-transitive of valency $\frac{q-1}{k}$.

Part 2. The group $M$ is transitive on $V$ and the sets $S_{i}:=\omega^{i-1}\left\langle\omega^{k}\right\rangle$, where $1 \leq i \leq k$, are the $M_{0}$-orbits in $V \backslash\{\boldsymbol{0}\}$. Since $\left\langle\omega^{k}\right\rangle=-\left\langle\omega^{k}\right\rangle$, it follows that, for each $i, S_{i}=-S_{i}$ and hence $S_{i}=\mathcal{O}_{i}(\mathbf{0})$ for some self-paired non-trivial $M$-orbital $\mathcal{O}_{i}$. Then by Lemmas 2.8 and $2.9, E_{i}=E\left(\mathcal{O}_{i}\right)$, and $\Gamma\left(\mathcal{O}_{i}\right)=\left(V, E_{i}\right)$ is $M$-arc-transitive for each $i$. Moreover $\operatorname{Cyc}(q, k)$ is a partition of $E K_{q}$, so $\left(K_{q}, \operatorname{Cyc}(q, k)\right)$ is a factorisation. Finally, since $\widehat{\omega} \in G_{0}$ maps $S_{i}$ to $S_{i+1}$ for $i=1, \ldots, k-1$, and $S_{k}$ to $S_{1}$, it follows from Lemma 2.4, and the fact that $M \triangleleft G$, that $G$ permutes the non-trivial $M$-orbitals transitively. Hence $\operatorname{Cyc}(q, k)$ is $G$-invariant, $\left(K_{q}, \operatorname{Cyc}(q, k)\right)$ is $(G, M)$ homogeneous, and $G$ induces isomorphisms between the $k$ factors $\Gamma\left(\mathcal{O}_{i}\right)$ so all are $M$-arc-transitive and isomorphic to $\Gamma\left(\mathcal{O}_{1}\right)=\operatorname{GPaley}\left(q, \frac{q-1}{k}\right)$. 
Finally in this subsection we characterise the examples arising from any affine 2-transitive group $G$ (not only the one-dimensional groups) in the case where $M_{0}$ is contained in the scalar subgroup of $\Gamma \mathrm{L}(a, q)$.

Lemma 4.3 Let $\left(K_{q^{a}}, \mathcal{E}\right)$ be an $(G, M)$-homogeneous arc transitive factorisation with $G, M$ as in Condition 3.5, and suppose that $\phi \in M_{0} \leq Z(\operatorname{GL}(a, q))$. Then $\mathcal{E}=$ $\operatorname{Cyc}\left(q^{a}, k\right)$ and we may replace $G$ by a 2-transitive one-dimensional affine group so that Theorem 1.1(2)(a) holds.

Proof Now $M_{0} \leq Z(\mathrm{GL}(a, q)) \leq \mathrm{GL}\left(1, q^{a}\right)$. Temporarily identify $V$ with $\mathbb{F}_{q^{a}}$, let $\omega$ be a primitive element of $\mathbb{F}_{q^{a}}$, and use the notation introduced above. Then $M_{0}=\left\langle\widehat{\omega}^{k}\right\rangle<\langle\widehat{\omega}\rangle=\operatorname{GL}\left(1, q^{a}\right)$, where $k$ divides $q^{a}-1$ and since $\phi \in M_{0}$, either $q$ or $\frac{q^{a}-1}{k}$ is even. Moreover the $M_{0}$ orbits in $V \backslash\{\boldsymbol{0}\}$ are the sets $S_{i}=-S_{i}=\omega^{i-1}\left\langle\omega^{k}\right\rangle$, for $i=1, \ldots, k$. Hence $\mathcal{E}$ is the cyclotomic partition $\operatorname{Cyc}\left(q^{a}, k\right)$. If $H=T \rtimes\langle\widehat{\omega}\rangle$ then $M \triangleleft H \leq \mathrm{A} \Gamma \mathrm{L}\left(1, q^{a}\right)$, and as in the proof of Proposition 4.2, $\left(K_{q^{a}}, \mathcal{E}\right)$ is an $(H, M)$-homogeneous arc-transitive factorisation. Replacing $G$ by $H$ we have that Theorem 1.1(2)(a) holds.

\subsection{Standard parameters for one-dimensional affine groups}

Foulser [10] gives a standard generating set for each subgroup of $\Gamma \mathrm{L}(1, q)=\langle\widehat{\omega}, \alpha\rangle$ that facilitates the checking of various important properties of the subgroups.

Lemma 4.4 [10, Lemma 4.1] Let $H \leq \Gamma \mathrm{L}\left(1, p^{R}\right)=\langle\widehat{\omega}, \alpha\rangle$. Then there exist unique integers $d$, e and s such that $H=\left\langle\widehat{\omega}^{d}, \widehat{\omega}^{e} \alpha^{s}\right\rangle$, and the following all hold:

(1) $d>0$ and $d \mid\left(p^{R}-1\right)$;

(2) $s>0$ and $s \mid R$;

(3) $0 \leq e<d$ and $e\left(p^{R}-1\right) /\left(p^{s}-1\right) \equiv 0(\bmod d)$.

Definition 4.5 (Standard Form) If $H=\left\langle\widehat{\omega}^{d}, \widehat{\omega}^{e} \alpha^{s}\right\rangle \leq \Gamma \mathrm{L}\left(1, p^{R}\right)$ and the integers $d, e$ and $s$ satisfy conditions (1)-(3) of Lemma 4.4, then the representation $H=$ $\left\langle\widehat{\omega}^{d}, \widehat{\omega}^{e} \alpha^{s}\right\rangle$ is said to be in standard form with standard parameters $(d, e, s)$.

Remark 4.6 In subsequent results (for example, see Lemmas 4.7-4.10), we will always work with subgroups of $\Gamma \mathrm{L}\left(1, p^{R}\right)$ given in standard form. To emphasise this we give an example: if $p=3$ then $H=\left\langle\widehat{\omega}^{3}, \alpha\right\rangle$ is not expressed in standard form since condition (1) of Lemma 4.4 fails. The standard form for this subgroup is $H=\langle\widehat{\omega}, \alpha\rangle$ and by Lemma 4.4, we know that this expression in standard form is unique.

First we give necessary and sufficient conditions on the standard parameters of a subgroup for it to be transitive on $V^{*}:=V \backslash\{\boldsymbol{0}\}$. This criteria may be found in [11, Section 3]. We provide a proof as we need the details later for determining the possibilities for $M_{0}$. 
Lemma 4.7 (Transitivity) Suppose $G_{0}=\left\langle\widehat{\omega}^{d}, \widehat{\omega}^{e} \alpha^{s}\right\rangle \leq \Gamma \mathrm{L}\left(1, p^{R}\right)$ is in standard form. Then $G_{0}$ is transitive on $V^{*}$ if and only if either $d=1$ (so $e=0$ ), or both of the following hold:

1. $e>0, d$ divides $e\left(\left(p^{d s}-1\right) /\left(p^{s}-1\right)\right)$, and

2. if $1<d^{\prime}<d$, then $d$ does not divide $e\left(\left(p^{d^{\prime} s}-1\right) /\left(p^{s}-1\right)\right)$.

Proof The set of orbits of $H:=\left\langle\widehat{\omega}^{d}\right\rangle$ in $V^{*}$ is $\Omega:=\left\{\left\langle\omega^{d}\right\rangle, \omega\left\langle\omega^{d}\right\rangle, \ldots, \omega^{d-1}\left\langle\omega^{d}\right\rangle\right\}$, and $\tau:=\widehat{\omega}^{e} \alpha^{s}$ induces a permutation of $\Omega$. Moreover, $G_{0}$ is transitive on $V^{*}$ if and only if $\langle\tau\rangle$ is transitive on $\Omega$. (To determine the image of $\omega^{i}\left\langle\omega^{d}\right\rangle$ under $\tau$, we simply need to find the "coset" of $\left\langle\omega^{d}\right\rangle$ containing $\left(\omega^{i}\right)^{\tau}$.)

If $e=0$ then $\tau=\alpha^{s}$, and since $\left(\widehat{\omega}^{d}\right)^{\alpha^{s}}=\widehat{\omega}^{d p^{s}} \in\left\langle\widehat{\omega}^{d}\right\rangle$, it follows that $\tau$ acts trivially on $\Omega$. Thus in this case $G_{0}$ is transitive on $V^{*}$ if and only if $d=1$. From now on suppose that $e \neq 0$. Then we have:

$\langle\tau\rangle$ is transitive on $\Omega \Longleftrightarrow(1) \tau^{d}$ fixes $\left\langle\omega^{d}\right\rangle$, and

(2) if $1 \leq d^{\prime}<d$, then $\tau^{d^{\prime}}$ does not fix $\left\langle\omega^{d}\right\rangle$

$\Longleftrightarrow(1) \omega^{e p^{s}\left(\left(p^{d s}-1\right) /\left(p^{s}-1\right)\right)} \in\left\langle\omega^{d}\right\rangle$, and

(2) if $1 \leq d^{\prime}<d$, then $\omega^{e p^{s}\left(\left(p^{d^{\prime} s}-1\right) /\left(p^{s}-1\right)\right)} \notin\left\langle\omega^{d}\right\rangle$

$\Longleftrightarrow(1) d$ divides $e p^{s}\left(\left(p^{d s}-1\right) /\left(p^{s}-1\right)\right)$, and

(2) if $1 \leq d^{\prime}<d$, then $d$ does not divide $e p^{s}\left(\frac{p^{d^{\prime} s}-1}{p^{s}-1}\right)$.

Since $d \mid\left(p^{R}-1\right)$ by Definition 4.5, it follows that $\operatorname{gcd}(p, d)=1$. So

$\langle\tau\rangle$ is transitive on $\Omega \Longleftrightarrow(1) d$ divides $e\left(\left(p^{d s}-1\right) /\left(p^{s}-1\right)\right)$, and

(2) if $1 \leq d^{\prime}<d$, then $d$ does not divide $e\left(\frac{p^{d^{\prime} s}-1}{p^{s}-1}\right)$.

We need to study normal subgroups $M_{0}$ in standard form of a given $G_{0}$ in standard form. Our next tasks are to give criteria for containment of one subgroup in another, and for normality.

Lemma 4.8 (Containment) Suppose $M_{0}=\left\langle\widehat{\omega}^{d_{1}}, \widehat{\omega}^{e_{1}} \alpha^{s_{1}}\right\rangle$ and $G_{0}=\left\langle\widehat{\omega}^{d}, \widehat{\omega}^{e}, \alpha\right\rangle$ are subgroups of $\Gamma \mathrm{L}\left(1, p^{R}\right)$ expressed in standard form. Then $M_{0}$ is a subgroup of $G_{0}$ if and only if

(1) $d \mid d_{1}$,

(2) $s \mid s_{1}$,

(3) and $d \mid\left(e \frac{\left(p^{s_{1}}-1\right)}{\left(p^{s}-1\right)}-e_{1}\right)$.

Proof Suppose $M_{0} \leq G_{0}$. Then $\widehat{\omega}^{d_{1}}$ and $\widehat{\omega}^{e_{1}} \alpha^{s_{1}}$ are elements of $G_{0}$. Let $B=\langle\widehat{\omega}\rangle$. Then $G_{0} \cap B=\left\langle\widehat{\omega}^{d}\right\rangle$ contains $M_{0} \cap B=\left\langle\widehat{\omega}^{d_{1}}\right\rangle$, so $\left|\left\langle\widehat{\omega}^{d_{1}}\right\rangle\right|$ divides $\left|\left\langle\widehat{\omega}^{d}\right\rangle\right|$ and hence 
$d \mid d_{1}$. Also, we have $M_{0} /\left(M_{0} \cap B\right) \cong M_{0} B / B \lesssim G_{0} B / B \cong G_{0} /\left(G_{0} \cap B\right)$. Since $M_{0} /\left(M_{0} \cap B\right)=\left\langle\left\langle\widehat{\omega}^{d_{1}}\right\rangle \widehat{\omega}^{e_{1}} \alpha^{s_{1}}\right\rangle \cong \mathbb{Z}_{R / s_{1}}$ and $G_{0} /\left(G_{0} \cap B\right)=\left\langle\left\langle\widehat{\omega}^{d}\right\rangle \widehat{\omega}^{e} \alpha^{s}\right\rangle \cong \mathbb{Z}_{R / s}$, it follows that $s \mid s_{1}$.

Given that $s$ divides $s_{1}$ (as shown above), we have $\left(\widehat{\omega}^{e} \alpha^{s}\right)^{s_{1} / s} \in G_{0}$. Now by [11, Lemma 2.1], for each $i \geq 1,\left(\widehat{\omega}^{e} \alpha^{s}\right)^{i}=\widehat{\omega}^{J} \alpha^{s i}$ where $J \equiv e\left(p^{s i}-1\right) /\left(p^{s}-1\right)(\bmod$ $\left.p^{R}-1\right)$, and hence

$$
\left(\widehat{\omega}^{e} \alpha^{s}\right)^{s_{1} / s}=\widehat{\omega}^{J^{\prime}} \alpha^{s_{1}},
$$

where $J^{\prime}=e\left(p^{s_{1}}-1\right) /\left(p^{s}-1\right)$. Writing

$$
\left(\widehat{\omega}^{e} \alpha^{s}\right)^{s_{1} / s}=\widehat{\omega}^{J^{\prime}} \alpha^{s_{1}}=\widehat{\omega}^{J^{\prime}} \widehat{\omega}^{-e_{1}} \widehat{\omega}^{e_{1}} \alpha^{s_{1}},
$$

and since $\left(\widehat{\omega}^{e} \alpha^{s}\right)^{s_{1} / s}, \widehat{\omega}^{e_{1}} \alpha^{s_{1}} \in G_{0}$, we see that $\widehat{\omega}^{J^{\prime}-e_{1}} \in G_{0}$ and this is true if and only if $d \mid\left(J^{\prime}-e_{1}\right)$.

Conversely, suppose the three conditions of the lemma are satisfied. Then since $d \mid d_{1}$, there exists an integer $j$ such that $\left(\widehat{\omega}^{d}\right)^{j}=\widehat{\omega}^{d_{1}}$. Thus $\widehat{\omega}^{d_{1}} \in G_{0}$. Now $s \mid s_{1}$ and $d \mid\left(e \frac{\left(p^{s_{1}}-1\right)}{\left(p^{s}-1\right)}-e_{1}\right)$. As above, by [11, Lemma 2.1], we get $\left(\widehat{\omega}^{e} \alpha^{s}\right)^{s_{1} / s} \in G_{0}$. However, we know that

$$
\left(\widehat{\omega}^{e} \alpha^{s}\right)^{s_{1} / s}=\widehat{\omega}^{J^{\prime}} \alpha^{s_{1}}=\widehat{\omega}^{J^{\prime}-e_{1}} \widehat{\omega}^{e_{1}} \alpha^{s_{1}} \in G_{0},
$$

where $J^{\prime}-e_{1}=\left(e \frac{\left(p^{s_{1}}-1\right)}{\left(p^{s}-1\right)}-e_{1}\right)$. Since $d \mid\left(J^{\prime}-e_{1}\right)$, we have $\widehat{\omega}^{J^{\prime}-e_{1}} \in G_{0}$, forcing $\widehat{\omega}^{e_{1}} \alpha^{s_{1}}$ to be in $G_{0}$.

Lemma 4.9 (Normality) Suppose $M_{0}=\left\langle\widehat{\omega}^{d_{1}}, \widehat{\omega}^{e_{1}} \alpha^{s_{1}}\right\rangle$ is in standard form and is a subgroup of $G_{0}=\left\langle\widehat{\omega}^{d}, \widehat{\omega}^{e} \alpha^{s}\right\rangle$ (so the conditions of Lemma 4.8 hold). Then $M_{0}$ is normal in $G_{0}$ if and only if

(1) $d_{1} \mid d\left(p^{s_{1}}-1\right)$ and

(2) $d_{1} \mid\left(e_{1}\left(p^{s}-1\right)+e p^{s}\left(p^{R-s_{1}}-1\right)\right)$.

Proof Now $M_{0}$ is normal in $G_{0}$ if and only if $\left(\widehat{\omega}^{d_{1}}\right)^{g} \in M_{0}$ and $\left(\widehat{\omega}^{e_{1}} \alpha^{s_{1}}\right)^{g} \in M_{0}$ for all $g \in G_{0}$. Since $\left\langle\widehat{\omega}^{d_{1}}\right\rangle \triangleleft G_{0}$ whenever $M_{0}=\left\langle\widehat{\omega}^{d_{1}}, \widehat{\omega}^{e_{1}} \alpha^{s_{1}}\right\rangle$ is a subgroup of $G_{0}$, it follows that $M_{0}$ is normal in $G_{0}$ if and only if $\left(\widehat{\omega}^{e_{1}} \alpha^{s_{1}}\right)^{g} \in M_{0}$ for all $g \in G_{0}$. Furthermore, since $G_{0}=\left\langle\widehat{\omega}^{d}, \widehat{\omega}^{e} \alpha^{s}\right\rangle$, we have that $M_{0}$ is normal in $G_{0}$ if and only if $\left(\widehat{\omega}^{e_{1}} \alpha^{s_{1}}\right)^{\widehat{\omega}^{d}} \in M_{0}$ and $\left(\widehat{\omega}^{e_{1}} \alpha^{s_{1}}\right)^{\widehat{\omega}^{e} \alpha^{s}} \in M_{0}$. Now

$$
\left(\widehat{\omega}^{e_{1}} \alpha^{s_{1}}\right)^{\widehat{\omega}^{d}}=\widehat{\omega}^{-d} \widehat{\omega}^{e_{1}} \alpha^{s_{1}} \widehat{\omega}^{d}=\widehat{\omega}^{e_{1}-d} \alpha^{s_{1}} \widehat{\omega}^{d}=\widehat{\omega}^{e_{1}} \alpha^{s_{1}} \widehat{\omega}^{d-d p^{s_{1}}}
$$

Thus

$$
\begin{aligned}
\left(\widehat{\omega}^{e_{1}} \alpha^{s_{1}}\right)^{\widehat{\omega}^{d}} \in M_{0} & \Longleftrightarrow \widehat{\omega}^{d-d p^{s_{1}}} \in M_{0} \\
& \Longleftrightarrow \widehat{\omega}^{d-d p^{s_{1}}} \in M_{0} \cap\langle\widehat{\omega}\rangle=\left\langle\widehat{\omega}^{d_{1}}\right\rangle \\
& \Longleftrightarrow d_{1} \mid d\left(p^{s_{1}}-1\right) .
\end{aligned}
$$


Next consider $\left(\widehat{\omega}^{e_{1}} \alpha^{s_{1}}\right)^{\widehat{\omega}^{e} \alpha^{s}}$. Then

$$
\begin{aligned}
& \left(\widehat{\omega}^{e_{1}} \alpha^{s_{1}}\right)^{\widehat{\omega}^{e} \alpha^{s}}=\left(\widehat{\omega}^{e_{1}-e} \alpha^{s_{1}} \widehat{\omega}^{e}\right)^{\alpha^{s}}=\widehat{\omega}^{\left(e_{1}-e\right) p^{s}}\left(\alpha^{s_{1}} \widehat{\omega}^{e}\right)^{\alpha^{s}}=\widehat{\omega}^{\left(e_{1}-e\right) p^{s}} \alpha^{s_{1}} \widehat{\omega}^{e p^{s}} \\
& =\widehat{\omega}^{\left(e_{1}-e\right) p^{s}} \alpha^{s_{1}} \widehat{\omega}^{e p^{s}}\left(\alpha^{s_{1}}\right)^{-1} \alpha^{s_{1}}=\widehat{\omega}^{\left(e_{1}-e\right) p^{s}}\left(\widehat{\omega}^{e p^{s}}\right)^{\left(\alpha^{s_{1}}\right)^{-1}} \alpha^{s_{1}} \\
& =\widehat{\omega}^{\left(e_{1}-e\right) p^{s}} \widehat{\omega}^{e p^{R-s_{1}+s}} \alpha^{s_{1}}=\widehat{\omega}^{\left(e_{1}-e\right) p^{s}+e p^{R-s_{1}+s}-e_{1}}\left(\widehat{\omega}^{e_{1}} \alpha^{s_{1}}\right) \text {. }
\end{aligned}
$$

Hence $\left(\widehat{\omega}^{e_{1}} \alpha^{s_{1}}\right)^{\widehat{\omega}^{e} \alpha^{s}} \in M_{0}$ if and only if $\widehat{\omega}^{\left(e_{1}-e\right) p^{s}+e p^{R-s_{1}+s}-e_{1}} \in M_{0}$, and

$$
\begin{aligned}
\widehat{\omega}^{\left(e_{1}-e\right) p^{s}+e p^{R-s_{1}+s}-e_{1}} \in M_{0} & \Longleftrightarrow \widehat{\omega}^{\left(e_{1}-e\right) p^{s}+e p^{R-s_{1}+s}-e_{1}} \in M_{0} \cap\langle\widehat{\omega}\rangle \\
& \Longleftrightarrow d_{1} \mid\left(\left(e_{1}-e\right) p^{s}+e p^{R-s_{1}+s}-e_{1}\right) \\
& \Longleftrightarrow d_{1} \mid\left(e_{1}\left(p^{s}-1\right)+e p^{s}\left(p^{R-s_{1}}-1\right)\right) .
\end{aligned}
$$

Thus $M_{0}$ is normal in $G_{0}$ if and only if (1) $d_{1} \mid d\left(p^{s_{1}}-1\right)$ and (2) $d_{1} \mid\left(e_{1}\left(p^{s}-1\right)+\right.$ $\left.e p^{s}\left(p^{R-s_{1}}-1\right)\right)$.

Next, for $M_{0}$ normal in a subgroup $G_{0}$, and $G_{0}$ transitive on $V^{*}$, we determine the number of $M_{0}$-orbits in terms its parameters.

Lemma 4.10 (Orbit Length) Let $M_{0}=\left\langle\widehat{\omega}^{d_{1}}, \widehat{\omega}^{e_{1}} \alpha^{s_{1}}\right\rangle$ and $G_{0}=\left\langle\widehat{\omega}^{d}, \widehat{\omega}^{e} \alpha^{s}\right\rangle$ be subgroups of $\Gamma \mathrm{L}\left(1, p^{R}\right)$ expressed in standard form. Suppose also that $M_{0}$ is a normal subgroup of $G_{0}$ and $G_{0}$ is transitive on $V^{*}$. Then $M_{0}$ has $t_{0}=d_{1} / c$ orbits of equal length $\left(p^{R}-1\right) / t_{0}$ in $V^{*}$, where if $e_{1}=0$ then $c=1$; and if $e_{1} \neq 0$, then $c$ is determined by:

(1) $d_{1} \mid e_{1}\left(p^{c s_{1}}-1\right) /\left(p^{s_{1}}-1\right)$ and

(2) $d_{1} \nmid e_{1}\left(p^{c^{\prime} s_{1}}-1\right) /\left(p^{s_{1}}-1\right)$ for $c^{\prime}<c$.

Note that conditions (1) and (2) above certainly define a positive integer $c \leq \frac{R}{s_{1}}$, since by Lemma 4.4 (3) applied to $M_{0}, d_{1}$ divides $e_{1} \frac{p^{R}-1}{p^{s_{1}}-1}$.

Proof Suppose first that $e_{1} \neq 0$.The set of $\left\langle\widehat{\omega}^{d_{1}}\right\rangle$-orbits in $V^{*}$ is $\Omega_{1}:=\left\{\left\langle\omega^{d_{1}}\right\rangle, \omega\left\langle\omega^{d_{1}}\right\rangle\right.$, $\left.\ldots, \omega^{d_{1}-1}\left\langle\omega^{d_{1}}\right\rangle\right\}$, each orbit having length $\left(p^{R}-1\right) / d_{1}$. Now $\left\langle\widehat{\omega}^{d_{1}}\right\rangle$ is characteristic in $\left\langle\widehat{\omega}^{d}\right\rangle$ (since any subgroup of a cyclic group is characteristic), and since $\left\langle\widehat{\omega}^{d}\right\rangle \triangleleft G_{0}$, we have $\left\langle\widehat{\omega}^{d_{1}}\right\rangle \triangleleft G_{0}$. Thus $\Omega_{1}$ is invariant under $G_{0}$ and $G_{0}$ is transitive on $\Omega_{1}$. In this action the normal subgroup $M_{0}$ of $G_{0}$ therefore has orbits of equal length in $\Omega_{1}$ (see [33, Theorem 10.3]). Moreover, since $\left\langle\widehat{\omega}^{d_{1}}\right\rangle$ acts trivially on $\Omega_{1}$, the group induced by $M_{0}$ on $\Omega_{1}$ is equal to the group induced by $\left\langle\tau_{1}\right\rangle$, where $\tau_{1}:=\widehat{\omega}^{e_{1}} \alpha^{s_{1}}$. Thus the $\left\langle\tau_{1}\right\rangle$-orbits in $\Omega_{1}$ have equal length $c$, where $c \mid d_{1}$ and the $M_{0}$-orbits in $V^{*}$ have equal length $\left(p^{R}-1\right) c / d_{1}$. Hence the following conditions hold for $c$.

(1) $\tau_{1}^{c}$ fixes $\left\langle\omega^{d_{1}}\right\rangle$ and

(2) if $c^{\prime}<c$, then $\tau_{1}^{c^{\prime}}$ does not fix $\left\langle\omega^{d_{1}}\right\rangle$.

Using similar arguments to those in the proof of Lemma 4.7 , 
The $M_{0}$-orbits in $V^{*}$ are of equal length $\left(p^{R}-1\right) c / d_{1} \Longleftrightarrow(1) d_{1} \mid e_{1} \frac{p^{c s_{1}}-1}{p^{s_{1}}-1}$, and

(2) $d_{1} \nmid e_{1} \frac{p^{c^{\prime} s_{1}-1}}{p^{s_{1}-1}}$

for $c^{\prime}<c$.

As remarked before the proof, these conditions determine $c$ uniquely. Now suppose $e_{1}=0$. Then $\alpha$ fixes each of the orbits of $\left\langle\widehat{\omega}^{d_{1}}\right\rangle$ in $V^{*}$ setwise, and hence $M_{0}$ has $t_{0}=d_{1}$ orbits of equal length $\left(p^{R}-1\right) / d_{1}$ in $V^{*}$.

\subsection{Proof of Theorem 1.1 for the one-dimensional affine case}

Recall that we have $G, M$ as in Condition 3.5 with $a=1$ and that we are assuming that $\left(K_{q}, \mathcal{E}\right)$ is a $(G, M)$-homogeneous arc-transitive factorisation, with factors $\Gamma_{i}=$ $\operatorname{Cay}\left(V, S_{i}\right)=\Gamma\left(\mathcal{O}_{i}\right)$ where $S_{i}=\mathcal{O}_{i}(\mathbf{0})=-S_{i}$ for $1 \leq i \leq k$. By Lemma 4.3, we may assume that $M_{0} \not \mathbf{G L}(1, q)$ where $n=q=p^{R}$.

Suppose that in standard form $G_{0}=\left\langle\widehat{\omega}^{d}, \widehat{\omega}^{e} \alpha^{s}\right\rangle$ and $M_{0}=\left\langle\widehat{\omega}^{d_{1}}, \widehat{\omega}^{e_{1}} \alpha^{s_{1}}\right\rangle$ with standard parameters $(d, e, s)$ and $\left(d_{1}, e_{1}, s_{1}\right)$ respectively. Since $M_{0} \not \leq \operatorname{GL}(1, q)$, the parameter $s_{1}>0$.

Lemma 4.11 If $e_{1}=0$ then $d_{1}=k$ and $\mathcal{E}(M)=\operatorname{Cyc}(q, k)$ as in Part 2(a) of Theorem 1.1 .

Proof If $e_{1}=0$ then by Lemma 4.10 all $M_{0}$-orbits in $V^{*}$ have length $\frac{q-1}{d_{1}}$, and hence $k=d_{1}$ and the $M_{0}$-orbits in $V^{*}$ coincide with the orbits of $\left\langle\widehat{\omega}^{k}\right\rangle$. It follows that $\mathcal{E}(M)=\operatorname{Cyc}(q, k)$.

Thus we may assume in addition that $e_{1} \neq 0$. Now since $G_{0}$ is transitive on $V^{*}$, $M_{0}$ is normal in $G_{0}$ and $M_{0}$ has $k$ orbits of length $\frac{q-1}{k}$ in $V^{*}$, the parameters satisfy a number of conditions given in the results of the previous subsection. We collect these conditions below and prove that they are sufficient for the existence of such a factorisation.

Condition 4.12 Let $M_{0}=\left\langle\widehat{\omega}^{d_{1}}, \widehat{\omega}^{e_{1}} \alpha^{s_{1}}\right\rangle$ and $G_{0}=\left\langle\widehat{\omega}^{d}, \widehat{\omega}^{e} \alpha^{s}\right\rangle$ be subgroups of $\Gamma L\left(1, p^{R}\right)=\langle\widehat{\omega}, \alpha\rangle$. Then $\left(G_{0}, M_{0}\right)$ is said to be admissible, and also the pair of integer triples $(d, e, s)$ and $\left(d_{1}, e_{1}, s_{1}\right)$ are said to be admissible, if $0<s<R, 0<$ $s_{1}<R, 0<e_{1}<d_{1}, 0 \leq e<d$ and the following all hold.
(1) $d_{1} \mid\left(p^{R}-1\right)$,
(2) $s_{1} \mid R$,
(3) $d_{1} \mid\left(e_{1} \frac{p^{R}-1}{p^{s_{1}}-1}\right)$,
(4) $d \mid d_{1}$,
(5) $s \mid s_{1}$,
(6) $d \mid\left(e \frac{\left(p^{s_{1}}-1\right)}{\left(p^{s}-1\right)}-e_{1}\right)$,
(7) $d_{1} \mid d\left(p^{s_{1}}-1\right)$ and
(8) $d_{1} \mid\left(e_{1}\left(p^{s}-1\right)+e p^{s}\left(p^{R-s_{1}}-1\right)\right)$. 
Furthermore, either $d=1$ and $e=0$, or both of the next two conditions hold:

(9) $e>0, d$ divides $e\left(\left(p^{d s}-1\right) /\left(p^{s}-1\right)\right)$, and

(10) if $1<d^{\prime}<d$, then $d$ does not divide $e\left(\left(p^{d^{\prime} s}-1\right) /\left(p^{s}-1\right)\right)$.

Finally, the positive integer $c$ determined by the next two conditions is strictly less than $d_{1}$ :

(11) $d_{1} \mid e_{1}\left(p^{c s_{1}}-1\right) /\left(p^{s_{1}}-1\right)$ and

(12) $d_{1} \nmid e_{1}\left(p^{c^{\prime} s_{1}}-1\right) /\left(p^{s_{1}}-1\right)$ for $c^{\prime}<c$.

Theorem 4.13 Let $M_{0}=\left\langle\widehat{\omega}^{d_{1}}, \widehat{\omega}^{e_{1}} \alpha^{s_{1}}\right\rangle$ and $G_{0}=\left\langle\widehat{\omega}^{d}, \widehat{\omega}^{e} \alpha^{s}\right\rangle$ be subgroups of $\Gamma \mathrm{L}\left(1, p^{R}\right)=\langle\widehat{\omega}, \alpha\rangle$ acting on $V^{*}$, such that $M_{0} \not \mathbf{G L}\left(1, p^{R}\right)$ and $\phi \in M_{0}$ (where $\phi: x \longrightarrow-x)$. Further let $G=T \rtimes G_{0}$ and $M=T \rtimes M_{0}$ be corresponding subgroups of $\mathrm{A} \Gamma \mathrm{L}\left(1, p^{R}\right)$, where $T=\mathbb{Z}_{p}^{R}$.

(1) If $\left(K_{p^{R}}, \mathcal{E}\right)$ is a $(G, M)$-homogeneous arc-transitive factorisation of index $k$, and if $(d, e, s)$ and $\left(d_{1}, e_{1}, s_{1}\right)$ are standard parameters for $G_{0}, M_{0}$ respectively, then $\left(G_{0}, M_{0}\right)$ is admissible and $k=d_{1} / c$ where $c$ is the integer determined by Condition 4.12(11), (12).

(2) Conversely if $(d, e, s),\left(d_{1}, e_{1}, s_{1}\right)$ is admissible, then $\left(K_{p^{R}}, \mathcal{E}(M)\right)$ is a $(G, M)$ homogeneous arc-transitive factorisation of index $k=d_{1} / c$, where $c$ is the integer determined by Condition 4.12(11), (12), and $\mathcal{E}(M)$ is as in Definition 3.2.

Proof Suppose that the hypotheses of Theorem 4.13(1) hold. We verify that all parts of Condition 4.12 hold. Parts (1), (2), (3) follow from Lemma 4.4, parts (4), (5), (6) from Lemma 4.8, parts (7), (8) from Lemma 4.9. By Lemma 4.7, either $(d, e)=(1,0)$ or conditions (9), (10) both hold. By Lemma 4.10, the integer $c$ determined by Condition 4.12(11), (12) divides $d_{1}$ and $d_{1} / c$ is the number of $M_{0}$-orbits in $V^{*}$. Since this number is $k$ it follows that $c<d_{1}$. Thus $\left(G_{0}, M_{0}\right)$ is admissible and Part 1 is proved.

Now suppose that the pair $(d, e, s),\left(d_{1}, e_{1}, s_{1}\right)$ is admissible, as in Part 2. By Condition 4.12(1)-(3), $\left(d_{1}, e_{1}, s_{1}\right)$ are standard generators for $M_{0}$. To see that $G_{0}$ is also in standard form, observe that Condition 4.12(1),(4) together imply that $d$ | $\left(p^{R}-1\right)$, while Condition 4.12(2),(5) imply that $s \mid R$. Finally by Condition 4.12(6), we have $d \mid\left(e \frac{\left(p^{s_{1}}-1\right)}{\left(p^{s}-1\right)}-e_{1}\right)$, and hence (multiplying by $\left(p^{R}-1\right) /\left(p^{s_{1}}-1\right)$, which is an integer by (2))

$$
d \text { divides } e\left(\frac{p^{R}-1}{p^{s}-1}\right)-e_{1}\left(\frac{p^{R}-1}{p^{s_{1}}-1}\right) .
$$

Now Condition 4.12(3),(4) imply that $d \mid e_{1}\left(\frac{p^{R}-1}{p^{s_{1}}-1}\right)$, and hence $d \mid e\left(p^{R}-1\right) /\left(p^{s}-\right.$ $1)$. Thus the three conditions of Lemma 4.4 are satisfied, so $G_{0}$ is in standard form. Next, Condition 4.12(4)-(6) and Lemma 4.8 imply that $M_{0} \leq G_{0}$, and then Condition 4.12(7), (8) and Lemma 4.9 imply that $M_{0}$ is a normal subgroup of $G_{0}$. Since either $(d, e)=(1,0)$ or Condition 4.12(9), (10) hold, it follows from Lemma 4.7 that $G_{0}$ is transitive on $V^{*}$. Finally by Lemma 4.10, the integer $c$ determined by Condition 4.12(11), (12) divides $d_{1}$ and $k:=d_{1} / c$ is the number of $M_{0}$-orbits in $V^{*}$. Since by assumption $c<d_{1}$, we have $k \geq 2$, and since $\phi \in M_{0}$ it follows from Lemma 2.9 that for each non-trivial $M$-orbital $\mathcal{O}$, the graph $\Gamma(\mathcal{O})$ is $M$-arc-transitive 
and $\mathcal{O}$ is selfpaired. Since $G_{0}$ is transitive on $V^{*}$, it permutes the $\Gamma(\mathcal{O})$ transitively by Lemma 2.4. Thus $\left(K_{p^{R}}, \mathcal{E}(M)\right)$ is a $(G, M)$-homogeneous arc-transitive factorisation with $\mathcal{E}(M)$ as in Definition 3.2.

Corollary 4.14 Let $\left(K_{q}, \mathcal{E}\right)$ be a $(G, M)$-homogeneous arc-transitive factorisation of index $k$ such that $G \leq \mathrm{A} \Gamma \mathrm{L}(1, q)$. Then Theorem 1.1(2)(a) or (b) holds.

Proof By Proposition 3.4, we may assume that $\phi \in M_{0}$. If $M_{0} \leq \mathrm{GL}(1, q)$ then by Lemma 4.3, Theorem 1.1(2)(a) holds, while if this is not the case then, by Theorem 4.13, Theorem 1.1(2)(b) holds.

\subsection{Twisted cyclotomic factorisations}

Even though Proposition 3.4 and Theorem 4.13 give a complete classification in terms of admissible pairs of the $(G, M)$-homogeneous arc-transitive factorisations of $K_{q}$ with $G$ a 2-transitive, one-dimensional affine group, it is not quite clear what pairs of subgroups $\left(G_{0}, M_{0}\right)$ are admissible according to Condition 4.12. An explicit classification of these admissible pairs would yield an explicit classification of the factorisations in Theorem 1.1(2)(b).

Problem 4.15 Give an explicit classification of admissible pairs $\left(G_{0}, M_{0}\right)$.

We construct an infinite family of admissible pairs $\left(G_{0}, M_{0}\right)$, demonstrating that the set is non-vacuous. First we give the corresonding $M$-edge-partition. As before $q=p^{R}$ and $\omega$ is a fixed primitive element of $\mathbb{F}_{q}$.

Definition 4.16 (Twisted generalised Paley graphs and twisted cyclotomic partitions) Let $R$ be even, $p \equiv 3(\bmod 4)$, and let $h$ be an odd divisor of $p-1$. Then the graph TGPaley $\left(p^{R}, \frac{p^{R}-1}{2 h}\right)=\operatorname{Cay}\left(V,\left\langle\omega^{4 h}\right\rangle \cup \omega^{3 h}\left\langle\omega^{4 h}\right\rangle\right)$ is called a twisted generalised $P a$ ley graph on $V$. The corresponding twisted cyclotomic partition of $K_{q}$ is the partition $\operatorname{TCyc}(q, 2 h)=\left\{E_{1}, \ldots, E_{2 h}\right\}$, where $E_{i}=\left\{\{u, v\} \mid v-u \in \omega^{2(i-1)}\left(\left\langle\omega^{4 h}\right\rangle \cup\right.\right.$ $\left.\left.\omega^{3 h}\left\langle\omega^{4 h}\right\rangle\right)\right\}$ for $1 \leq i \leq 2 h$.

Remark 4.17 Note that when $p$ is odd and $R$ is even, $8 \mid\left(p^{R}-1\right)$, and hence $\left\langle\omega^{4 h}\right\rangle=$ $-\left\langle\omega^{4 h}\right\rangle$ so the graph TGPaley $\left(q, \frac{q-1}{2 h}\right)$ is well defined as an undirected Cayley graph. In 2001, Peisert [27] classified all self-complementary arc-transitive graphs, proving that there are two infinite families of examples, namely the Paley graphs and the graphs TGPaley $\left(p^{R}, \frac{p^{R}-1}{2}\right)$. Furthermore, he proved (see [27, Lemma 6.4]) that, apart from the exceptional isomorphism $\operatorname{GPaley}(9,4) \cong \operatorname{TGPaley}(9,4)$, the graphs $\operatorname{TGPaley}\left(q, \frac{q-1}{2}\right)$ and $\operatorname{GPaley}\left(q, \frac{q-1}{2}\right)$ are not isomorphic. We extend this result below in Proposition 4.18 to show that the generalised Paley graphs and twisted generalised Paley graphs are not isomorphic apart from this one exception. (Note that, by considering the order and valency of $\operatorname{TGPaley}\left(p^{R}, \frac{p^{R}-1}{2 h}\right)$, the only generalised Paley graph it could possibly be isomorphic to would be $\operatorname{GPaley}\left(p^{R}, \frac{p^{R}-1}{2 h}\right)$.) Also in Proposition 4.18 we prove that $\left(K_{q}, \operatorname{TCyc}(q, 2 h)\right)$ is a homogeneous factorisation, called a twisted cyclotomic factorisation. 
Proposition 4.18 Let $R$ be even, $p \equiv 3(\bmod 4)$, and let $h$ be an odd divisor of $p-1$. Let $\Gamma=$ TGPaley $\left(p^{R}, \frac{p^{R}-1}{2 h}\right)$, let $G_{0}=\left\langle\widehat{\omega}^{2}, \widehat{\omega} \alpha\right\rangle$ and $M_{0}=\left\langle\widehat{\omega}^{4 h}, \widehat{\omega}^{h} \alpha\right\rangle$, subgroups of $\Gamma \mathrm{L}\left(1, p^{R}\right)$, with corresponding subgroups $G=T \rtimes G_{0}$ and $M=T \rtimes M_{0}$ of $\mathrm{A} \Gamma \mathrm{L}\left(1, p^{R}\right)$. Then

(1) $M \leq \operatorname{Aut}(\Gamma)$ and $\Gamma$ is $M$-arc-transitive; moreover either $(R, p, h)=(2,3,1)$ or the graphs $\Gamma$ and $\operatorname{GPaley}\left(p^{R}, \frac{p^{R}-1}{2 h}\right)$ are not isomorphic.

(2) $\left(G_{0}, M_{0}\right)$ is admissible and the integer c determined in Condition 4.12(10), (11) is 2 .

(3) $\left(K_{p^{R}}, \operatorname{TCyc}(q, 2 h)\right)$ is a $(G, M)$-homogeneous arc-transitive factorisation of index $2 h$, with all factors isomorphic to $\Gamma$.

Proof (1) By definition, $\Gamma=\operatorname{Cay}(V, S)$, where $S=\left\langle\omega^{4 h}\right\rangle \cup \omega^{3 h}\left\langle\omega^{4 h}\right\rangle$. The $M_{0}$-orbit in $V$ containing $\omega^{4 h}$ is equal to $S$, since $\widehat{\omega}^{h} \alpha$ maps $\omega^{4 h}$ to $\omega^{5 h p}=\omega^{4 h p} . \omega^{h(p-3)} . \omega^{3 h} \in$ $\omega^{3 h}\left\langle\omega^{4 h}\right\rangle$. Thus $M \leq \operatorname{Aut}(\Gamma)$, and moreover, since $M$ is transitive and $M_{0}$ is transitive on $S$, it follows that $\Gamma$ is $M$-arc-transitive.

Suppose now that $(R, p, h) \neq(2,3,1)$, set $\Gamma^{\prime}=\operatorname{GPaley}\left(p^{r}, \frac{p^{R}-1}{2 h}\right)$, and suppose that $\sigma: \Gamma^{\prime} \longrightarrow \Gamma$ is an isomorphism. Now $\sigma \in \operatorname{Sym}(V)$ and conjugates $\operatorname{Aut}\left(\Gamma^{\prime}\right)$ to $\operatorname{Aut}(\Gamma)$. Moreover, as $\Gamma$ is arc-transitive we may assume that $\sigma$ fixes $\mathbf{0}$ and $\omega^{4 h}$. By [25, Theorem 1.3], Aut $\left(\Gamma^{\prime}\right)=T \rtimes L_{0} \leq \mathrm{A} \Gamma \mathrm{L}\left(1, p^{R}\right)$, where $L_{0}=\left\langle\widehat{\omega}^{2 h}, \alpha\right\rangle$. This implies in particular that $\sigma \in N_{\operatorname{Sym}(V)}(T)=\operatorname{AGL}(R, p)$, and since $\Sigma$ fixes $\mathbf{0}$, that $\sigma \in \mathrm{GL}(R, p)$. Suppose first that there exists a prime divisor $r$ of $p^{R}-1$ such that $r$ does not divide $p^{i}-1$ for any $i<R$. Then the unique subgroup $K \cong \mathbb{Z}_{r}$ of $L_{0}$ also lies in $M_{0}$ and acts irreducibly on $V$. It follows that $\sigma$ lies in $N_{\mathrm{GL}(R, p)}(K)=\langle\widehat{\omega}, \alpha\rangle$ (see for example, [15, Satz II.7.3]). A straightforward computation shows that $L_{0}$ is normal in $\langle\widehat{\omega}, \alpha\rangle$, and so $L_{0}^{\sigma}=L_{0}$ contains $M_{0}$, and this is a contradiction.

Thus no such prime exists and so, by a result of Zsigmondy [35], the even integer $R$ is 2 and $p=2^{a}-1$ for some $a$. By our assumption $a \geq 3$. We claim that $K:=\left\langle\widehat{\omega}^{4 h}\right\rangle$ is the unique cyclic subgroup of $L_{0}$ of index 4 . Let $K^{\prime}$ be such a subgroup. Since $\left|L_{0}:\left\langle\widehat{\omega}^{2 h}\right\rangle\right|=2$ it follows that $\left|K^{\prime}:\left(K^{\prime} \cap\left\langle\widehat{\omega}^{2 h}\right\rangle\right)\right| \leq 2$ and hence $K^{\prime}$ contains the unique subgroup $K_{0}=\left\langle\widehat{\omega}^{8 h}\right\rangle$ of $\left\langle\widehat{\omega}^{2 h}\right\rangle$ of order $\frac{\left|K^{\prime}\right|}{2}$. Now $L_{0} / K_{0} \cong D_{8}$ contains $K^{\prime} / K_{0}$ as a subgroup of order 2 , so either $K^{\prime}=K$ and the claim holds, or $K^{\prime}=$ $\left\langle\widehat{\omega}^{8 h}, \alpha \widehat{\omega}^{2 i}\right\rangle$ for some $i \in\{0,1,2,3\}$. Suppose we are in the latter case. As $K^{\prime}$ is abelian, $\widehat{\omega}^{8 h}=\left(\widehat{\omega}^{8 h}\right)^{\alpha \widehat{\omega}^{2 i}}=\widehat{\omega}^{8 h p}$. Since $2 h \mid(p-1)$, this implies that $p=7$ and so $h=1$ or 3 and $K^{\prime} \cong \mathbb{Z}_{12}$ or $\mathbb{Z}_{4}$ respectively. Now $\left(\alpha \widehat{\omega}^{2 i}\right)^{2}=\widehat{\omega}^{2 i(p+1)}=\widehat{\omega}^{16 i}$, and hence $\alpha \widehat{\omega}^{2 i}$ has order 2 (if $i=0$ or 3 ) or 6 (if $i=1$ or 2 ). In either case $K^{\prime}$ is not cyclic, which is a contradiction. Thus the claim is proved. Therefore $K^{\sigma}$ is the unique cyclic subgroup of $L_{0}^{\sigma}$ of index 4 . However the subgroup $\left\langle\widehat{\omega}^{4 h}\right\rangle$ of $M_{0}$ is such a subgroup. Hence $\sigma$ normalises $\left\langle\widehat{\omega}^{4 h}\right\rangle$. Moreover, $\widehat{\omega}^{4 h}$ acts irreducibly on $V$, and the argument at the end of the previous paragraph leads to a contradiction in this case also. Thus the graphs $\Gamma, \Gamma^{\prime}$ are not isomorphic when $(R, p, h) \neq(2,3,1)$. This completes the proof of Part 1.

(2) Using the facts that $h$ is odd, $R$ is even, $h \mid(p-1)$, and $p \equiv 3 \quad(\bmod 4)$, it is straightforward to verify each part of Condition 4.12 with the triples $(d, e, s)=$ $(2,1,1)$ and $\left(d_{1}, e_{1}, s_{1}\right)=(4 h, h, 1)$, and to determine that the positive integer $c$ is equal to 2 . 
(3) By Theorem 4.13(2), noting that $\phi \in M_{0}$, it follows that $\left(K_{p^{R}}, \mathcal{E}(M)\right)$ is a $(G, M)$-homogeneous arc-transitive factorisation. Moreover $G_{0}$ is transitive on $V^{*}$ and $G$ normalises $M$, and hence $G$ permutes the non-trivial $M$-orbitals transitively. Thus each non-trivial $M$-orbital $\mathcal{O}$ is selfpaired and the subset $\mathcal{O}(\mathbf{0})$ is equal to $\omega^{2 i} S$ for some $i$. It follows that $\mathcal{E}(M)=\operatorname{TCyc}(q, 2 h)$ and the index of $\left(K_{p^{R}}, \mathcal{E}(M)\right)$ is $2 h$.

To conclude, the generalised Paley graphs and twisted generalised Paley graphs are not in general isomorphic, and hence the cyclotomic factorisations and twisted cycloctomic factorisations form two infinite and different families of homogeneous arc-transitive factorisations of complete graphs. It would be interesting to know if there are any other infinite families of examples arising from the admissible pairs of Condition 4.12.

\section{The affine 2-transitive case}

In this section we complete the proof of Theorem 1.1. By Proposition 3.4, we may assume that $G, M$ are affine groups as in Condition 3.5 with $G$ acting 2-transitively on $V$, and $\mathcal{O}_{i}, E_{i}, \Gamma_{i}=\operatorname{Cay}\left(V, S_{i}\right)$ as given in the discussion following Condition 3.5. Moreover, by Corollary 4.14 we may assume that $G_{0}$ satisfies one of (b)-(h) of Theorem 2.5(2), and by Lemma 4.3 we may assume that $M_{0}$ is not contained in the subgroup of scalars of $\Gamma \mathrm{L}(a, q)$. First we show that cases (b)-(f) do not lead to any examples. Let $Z:=Z(G L(a, q))$, and for a subgroup $H$ of $\Gamma \mathrm{L}(a, q)$ let $\bar{H}$ denote the subgroup $H Z / Z$ of $\Gamma \mathrm{L}(a, q) / Z \cong \mathrm{P} \Gamma \mathrm{L}(a, q)$.

Lemma 5.1 The group $G_{0}$ satisfies one of $(g)$ or $(h)$ of Theorem 2.5(2).

Proof Assume that $G_{0}$ satisfies one of (b) to (f) of Theorem 2.5(2). Since $M_{0} \not \leq Z$ it follows that $\overline{M_{0}}$ is a non-trivial normal subgroup of $\overline{G_{0}}$. If case (b) holds for $G_{0}$, then one of (i) $\overline{M_{0}}$ contains $\operatorname{PSL}(a, q)$ so $M_{0}$ contains $\operatorname{SL}(a, q)$, or (ii) $(a, q)=(a, 2)$ and $M_{0}$ contains $\mathbb{Z}_{3}$, or (iii) $(a, q)=(a, 3)$ and $M_{0}$ contains the normal subgroup $Q_{8}$ of $G_{0}$. In each of these cases $M_{0}$ is transitive on $V^{*}$ which is a contradiction. Similarly if (c) or (d) holds then $M_{0}$ contains $\operatorname{Sp}(a, q)^{\prime}$ or $\mathrm{G}_{2}(q)^{\prime}$ (with $a=6$ ) respectively, and again $M_{0}$ is transitive on $V^{*}$, a contradiction. Finally in cases (e) and (f) the only non-scalar normal subgroup $M_{0}$ of $G_{0}$ is $G_{0}$ itself, again a contradiction.

\subsection{Case $2(g)$}

First we determine the possibilities for $M_{0}$. Here $V=V(2, q)$ and we let $P_{1}(V)$ denote the set of 1-spaces of $V$. Assertions regarding numbers and lengths of orbits of various subgroups on $P_{1}(V)$ were checked using MAGMA [8].

Lemma 5.2 Suppose that $G_{0}$ satisfies ( $g$ ) of Theorem 2.5(2) and $\phi \in M_{0}, M_{0} \not \leq Z$. Then one of the following holds. 
Table $1 M_{0}$-orbits on the 1-spaces of $V=V(2, q)$ where $M_{0}$ is as in Lemma 5.2(2)

\begin{tabular}{llll}
\hline$q$ & $M_{0}$ & $\begin{array}{l}\text { No. of } M_{0} \text {-orbits } \\
\text { in } P_{1}(V)\end{array}$ & $\begin{array}{l}\text { Length of each } M_{0} \text {-orbit } \\
\text { in } P_{1}(V)\end{array}$ \\
\hline 5 & 3 & 2 \\
7 & $Q_{8} \leq M_{0} \leq Z \circ Q_{8}$ & 2 & 4 \\
11 & & 3 & 4 \\
23 & 6 & 4 \\
7 & 2 & 4 \\
11 & & 1 & 12 \\
23 & 2 & 12 \\
7,11, & 1 & $\left|P_{1}(V)\right|$ \\
23 & $\overline{M_{0}}=\operatorname{PSL}(2,3)$ & & \\
\hline
\end{tabular}

(1) $\operatorname{SL}(2,5) \leq M_{0} \triangleleft G_{0} \leq N:=N_{\Gamma \mathrm{L}(2, q)}(\mathrm{SL}(2,5)), M_{0}$ is transitive on $P_{1}(V)$, and $q=9,19,29$ or 59. Moreover, either $\overline{G_{0}}=\overline{M_{0}} \cong A_{5}$, or $q=9$ and $\overline{G_{0}} \cong S_{5}$.

(2) $Q_{8} \leq M_{0} \triangleleft G_{0} \leq N:=N_{\Gamma L(2, q)}(\operatorname{SL}(2,3))$, and $q=5,7,11$ or 23. Moreover, either $M_{0} \leq Z \circ Q_{8}$, or $q \neq 5$ and $M_{0} \geq \mathrm{SL}(2,3)$; the $M_{0}$-orbits in $P_{1}(V)$ are described in Table 1. Also either $\overline{G_{0}} \cong \operatorname{PGL}(2,3)$, or $q=5,11$ and $\overline{G_{0}} \cong$ $\operatorname{PSL}(2,3)$.

Proof (1) Suppose first that SL(2, 5) $\unlhd G_{0} \leq \Gamma \mathrm{L}(2, q)$ with $q=9,11,19,29$ or 59. Then $G_{0} \leq N:=N_{\Gamma L(2, q)}(S L(2,5))$. and $A_{5} \cong \operatorname{PSL}(2,5) \unlhd \overline{G_{0}} \leq \bar{N}$. By [31, p. 417 (Ex. 7)], the subgroup $A_{5}$ is maximal in $\operatorname{PSL}(2, q)$ for each of these values of $q$, and there are two conjugacy classes of such subgroups interchanged by $\operatorname{PGL}(2, q)$. Thus $\bar{N}=\overline{G_{0}}=A_{5}$ for $q=11,19,29$ or 59 . For $q=9, \bar{N} \cong S_{5}$. Since $\overline{M_{0}}$ is a non-trivial normal subgroup of $\overline{G_{0}}, M_{0}$ must contain $\operatorname{SL}(2,5)$. In particular, $M_{0}$ is transitive on $P_{1}(V)$. If $q=11$ then $\operatorname{SL}(2,5)$ is transitive on $V^{*}$ and hence $q \neq 11$.

(2) Now suppose that $\operatorname{SL}(2,3) \unlhd G_{0} \leq \Gamma \mathrm{L}(2, q)$ with $q=5,7,11$ or 23 . Then the group $G_{0} \leq N:=N_{\Gamma \mathrm{L}(2, q)}(S L(2,3))$. By [31, Theorem 6.26(ii)], it follows that $\bar{N}=$ $\operatorname{PGL}(2,3) \cong S_{4}$, and so either $\overline{G_{0}}=\operatorname{PGL}(2,3)$, or $q=5,11$ and $\overline{G_{0}}=\operatorname{PSL}(2,3)$ (since PSL $(2,3)$ is transitive on the $P_{1}(V)$ only for $q=5$ or 11$)$. Since $\overline{M_{0}}$ is a nontrivial normal subgroup of $\overline{G_{0}}, M_{0}$ must contain $Q_{8}$ and hence either $M_{0} \leq Z \circ Q_{8}$ or $\operatorname{SL}(2,3) \leq M_{0}$. For each possibility for $M_{0}$, its orbits in $P_{1}(V)$ were computed using Magma and the results are given in Table 1. In the case where $q=5$ and $M_{0} \geq \operatorname{SL}(2,3)$, the group $M_{0}$ is transitive on $V^{*}$, which is not allowed. Thus for $q=5$, we can only have $Q_{8} \leq M_{0} \leq Z \circ Q_{8}$.

Although the information in Table 1 does not tell us much about the number of $M_{0}$-orbits in $V^{*}$, it is useful in enabling us to see (almost directly) if the resulting $M$-arc-transitive factors are connected.

Corollary 5.3 If $G_{0}, M_{0}$ are as in Lemma 5.2, then all of the $\Gamma_{i}=\mathrm{Cay}\left(V, S_{i}\right)$ are connected. 
Proof By Lemma 5.2, each $M_{0}$-orbit in $P_{1}(V)$ has size at least two, and so each $M_{0}$-orbit in $V^{*}$ contains a basis for $V=V(2, q)$. It follows that the Cayley graphs $\Gamma_{i}=\operatorname{Cay}\left(V, S_{i}\right)$ are connected.

Using Magma [8], we constructed explicitly all the possibilities for $M_{0}$ in Lemma 5.2(1), and in each line of Table 1, and we computed the number $k$ of $M_{0^{-}}$ orbits in $V^{*}$. Also, for each group $M=T \rtimes M_{0}$ we constructed the $M$-arc-transitive graph $\Gamma:=\Gamma(\mathcal{O})$, where $\mathcal{O}$ is the (selfpaired) $M$-orbital containing $(\mathbf{0}, v)$ for a fixed $v \in V^{*}$, and we computed $\operatorname{Aut}(\Gamma)$. The results are given in Tables 2 and 3 . Note that $\mathcal{O}$ is selfpaired since $\phi \in M_{0}$, and the graph $\Gamma$ has valency $\left(q^{2}-1\right) / k=|\mathcal{O}(\mathbf{0})|$. Also, since $M_{0}$ is normal in $G_{0}$ and $G_{0}$ is transitive on $V^{*},\left(K_{q^{2}}, \mathcal{E}(M)\right)$ is a $(G, M)$ homogeneous arc-transitive factorisation with all factors isomorphic to $\Gamma$. We make a formal definition of this graph $\Gamma$ as $G\left(q^{2}, k\right)$ in Definition 5.4, and a formal statement in Proposition 5.5 of the classification of this family of factorisations.

Definition 5.4 (The graph $G\left(q^{2}, k\right)$ ) Let $M=T \rtimes M_{0} \leq \mathrm{A} \Gamma \mathrm{L}(2, q)$ be an affine permutation group on $V=V(2, q)$ such that $M_{0}$ is one of the groups listed in Tables 2 and 3. Let $v$ be a fixed element of $V^{*}=V \backslash\{\boldsymbol{0}\}$, let $S:=v^{M_{0}}$, and let $k:=\left(q^{2}-1\right) /|S|$ (the number of $M_{0}$-orbits in $\left.V^{*}\right)$. Then $G\left(q^{2}, k\right)$ is defined as $\operatorname{Cay}(V, S)$.

Proposition 5.5 Let $\left(K_{q^{2}}, \mathcal{E}\right)$ be a $(G, M)$-homogeneous arc-transitive factorisation of index $k$ such that $G=T \rtimes G_{0}$, with $G_{0}$ as in Theorem $2.5(2)(g), M_{0} \not Z Z$ and $\phi \in$ $M_{0}$. Then $\mathcal{E}=\mathcal{E}(M)$, and all possibilities for $M_{0}, k$, the factors $\Gamma_{i} \cong G\left(q^{2}, k\right)$ and their valencies and automorphism groups are listed in Table 2 and 3. In particular, Theorem 1.1(2)(c) holds.

The proof follows from the discussion above and the MAGMA computations described. In Remark 5.7 we make a a series of comments about important aspects of these factorisations. First we determine precisely which of the examples also arise in other parts of Theorem 1.1.

Lemma 5.6 Let $\left(K_{q^{2}}, \mathcal{E}(M)\right)$ be a $(G, M)$-homogeneous arc-transitive factorisation corresponding to a line of Table 2 or 3 , and suppose that this factorisation also occurs in another Part of Theorem 1.1. Then either line (1) of Table 2 holds, or one of the lines (2) or (3) of Table 3 holds, and in each case $\mathcal{E}(M)=\operatorname{TCyc}\left(q^{2}, k\right)$ occurs in Part 2(b).

Proof The cases where $k=2$ were considered by Peisert in [27]. They are as follows.

(1) $q=9, \operatorname{SL}(2,5) \leq M_{0}<(Z \circ \operatorname{SL}(2,5)) \cdot \mathbb{Z}_{2}$ and $\Gamma_{i} \cong G\left(9^{2}, 2\right)$ (line (1) of Table 2),

(2) $q=7, \operatorname{SL}(2,3) \leq M_{0} \leq Z \circ \operatorname{SL}(2,3)$ or $M_{0}=Z \circ Q_{8}$, and $\Gamma_{i} \cong G\left(7^{2}, 2\right)$ (line (3) of Table 3), or

(3) $q=23, M_{0}=Z \circ \operatorname{SL}(2,3)$ and $\Gamma_{i} \cong G\left(23^{2}, 2\right)$ (line (11) of Table 3).

The $\Gamma_{i}$ in these cases are three exceptional arc-transitive self-complementary graphs denoted by Peisert as $G\left(9^{2}\right), G\left(7^{2}\right)$, and $G\left(23^{2}\right)$ respectively. By [27, Lemma 
Table $2 \operatorname{SL}(2,5) \leq M_{0} \triangleleft G_{0} \leq \Gamma \mathrm{L}(2, q)$, and $\operatorname{Aut}\left(G\left(q^{2}, k\right)\right)=T \rtimes(Y \circ \operatorname{SL}(2,5))$ (if $q=19,29$ or 59) or $T \rtimes\left((Y \circ \operatorname{SL}(2,5)) \cdot \mathbb{Z}_{2}\right)$ (if $\left.q=9\right)$, where $Y<Z=Z(\mathrm{GL}(2, q))$; for lines (2)-(11), Aut $\left(G\left(q^{2}, k\right)\right) \leq$ $\mathrm{A} \Gamma \mathrm{L}(2, q)$

\begin{tabular}{|c|c|c|c|c|c|c|}
\hline & $G\left(q^{2}, k\right)$ & $M_{0}$ & $\left|M_{0}\right|$ & $\frac{\left|V^{*}\right|}{k}$ & $\begin{array}{l}|Y|, \\
\text { where } \\
Y<Z\end{array}$ & Remarks \\
\hline \multirow{4}{*}{ (1) } & & $\operatorname{SL}(2,5)$ & 120 & & & TGPaley \\
\hline & $G\left(9^{2}, 2\right)$ & $Y \circ \operatorname{SL}(2,5)$ & 240 & 40 & 4 & see Remark 5.7(3) \\
\hline & & $(Y \circ \operatorname{SL}(2,5)) \cdot \mathbb{Z}_{2}$ & 480 & & & \\
\hline & & $\operatorname{SL}(2,5)$ & 120 & & & see Remark 5.7(4) \\
\hline \multirow[t]{3}{*}{ (2) } & $G\left(19^{2}, 3\right)$ & & & 120 & 6 & \\
\hline & & $Y \circ \operatorname{SL}(2,5)$ & 360 & & & \\
\hline & & $\operatorname{SL}(2,5)$ & 120 & & & see Remark 5.7(4) \\
\hline \multirow[t]{3}{*}{ (3) } & $G\left(29^{2}, 7\right)$ & & & 120 & 4 & \\
\hline & & $Y \circ \operatorname{SL}(2,5)$ & 240 & & & \\
\hline & & & & & & see Remark 5.7(4) \\
\hline (4) & $G\left(59^{2}, 29\right)$ & $\operatorname{SL}(2,5)$ & 120 & 120 & 1 & \\
\hline
\end{tabular}

6.6 and 6.7], $G\left(9^{2}, 2\right)$ and $G\left(7^{2}, 2\right)$ are isomorphic to the twisted generalised Paley graphs TGPaley $(81,40)$ and TGPaley $(49,24)$ respectively. Since $k=2$ this means that the edge-partition $\mathcal{E}(M)$ in these two cases is $\operatorname{TCyc}\left(9^{2}, 2\right)$ and $\operatorname{TCyc}\left(7^{2}, 2\right)$ respectively, as in Lemma 5.6(1). On the other hand, the graph $G\left(23^{2}, 2\right)$ is "new" in the sense that it is neither the Paley graph nor the twisted $\operatorname{TCyc}\left(23^{2}, 2\right)$, see [27, Lemma 6.8]. We show below that this case is really new.

In line (1) of Table 3, we verified using MAGMA that the factor $G(25,3)$ is a generalised Paley graph of valency 8 . Suppose that $\left(K_{25}, \mathcal{E}(M)\right)$ is also an $(H, L)$ homogeneous arc-transitive factorisation with $H=T \rtimes H_{0}, L=T \rtimes L_{0}, L_{0}<H_{0} \leq$ $\langle\widehat{\omega}, \alpha\rangle$. Since $G(25,3)$ is $L$-arc-transitive of valency $8, L_{0}$ must contain $\left\langle\widehat{\omega}^{6}\right\rangle$, and since $H_{0}$ permutes transitively on the 3 factors, it follows that either $H_{0}$ contains $\left\langle\widehat{\omega}^{2}\right\rangle$, or $L_{0} \leq\langle\widehat{\omega}\rangle$. In the latter case $\mathcal{E}(M)=\operatorname{Cyc}(25,3)$ by Lemma 4.3 , and we may therefore assume in this case that $H_{0}=\langle\widehat{\omega}, \alpha\rangle$. Thus the partition $\mathcal{E}(M)$ is preserved both by the subgroup $G_{0}$ that projects onto $\overline{G_{0}} \cong A_{4}$ or $S_{4}$ modulo $Z$, and also by $H_{0}$ that projects to $D_{12}$. These two subgroups generate the whole group $\operatorname{PGL}(2,5)$ which cannot act transitively on the three parts of Cyc $(25,3)$, a contradiction. Thus $L_{0} \not \leq$ $\langle\widehat{\omega}\rangle,\left\langle\widehat{\omega}^{2}\right\rangle \leq H_{0}$, and $\mathcal{E}(M) \neq \operatorname{Cyc}(25,3)$. We may assume that $(H, L)$ is admissible as in Condition 4.12. Let $H_{0}=\left\langle\widehat{\omega}^{d}, \widehat{\omega}^{e} \alpha^{s}\right\rangle$ and $L_{0}=\left\langle\widehat{\omega}^{d_{1}}, \widehat{\omega}^{e_{i}} \alpha^{s_{1}}\right\rangle$ in standard form. From our discussion we must have $s_{1}=s=1, d \leq 2, d_{1} \mid 6$, and by Lemma 4.11, $0<e_{1}<d_{1}$. Now Aut(GPaley $\left.(25,8)\right) \cap \mathrm{A} \Gamma \mathrm{L}(1,25)=\left\langle\widehat{\omega}^{3}, \alpha\right\rangle$, and since $L$ acts arctransitively, $d_{1} \mid 6$ and hence $e_{1}=3, d_{1}=6$. By Condition 4.12(7), 6| $4 d$ so $d$ is a multiple of 3 , which contradicts the fact that $d \leq 2$. Thus $\mathcal{E}(M)$ for line (1) of Table 3 does not occur in any other part of Theorem 1.1 .

In line (2) of Table $3, G(49,6) \cong$ TPaley $(49,8)$, there are 6 factors, so considering the 2-part of $|G|$ we see that $G_{0}$ must project onto $\overline{G_{0}}=S_{4}$ modulo $Z$. Suppose 
Table $3 M_{0} \supseteq \operatorname{SL}(2,3)$ or $M_{0} \supseteq Q_{8}, M_{0} \triangleleft G_{0} \leq \mathrm{GL}(2, q)$ and $T=\mathbb{Z}_{q}^{2}$, where $q=5,7,11$ or 23

\begin{tabular}{|c|c|c|c|c|c|c|}
\hline & $G\left(q^{2}, k\right)$ & $M_{0}$ & $\left|M_{0}\right|$ & $\left|V^{*}\right| / k$ & $\operatorname{Aut}\left(G\left(q^{2}, k\right)\right)$ & Remarks \\
\hline \multirow[t]{2}{*}{ (1) } & $G\left(5^{2}, 3\right)$ & $Q_{8}$ & 8 & 8 & $S_{5} ? S_{2}$ & GPaley \\
\hline & & $Z \circ Q_{8}$ & 16 & & (in product action) & see Remark 5.7(5) \\
\hline \multirow[t]{3}{*}{ (2) } & $G\left(7^{2}, 6\right)$ & $Q_{8}$ & 8 & 8 & $T \rtimes\left(Q_{8} \cdot \mathbb{Z}_{3}\right)$ & TGPaley \\
\hline & & $Q_{8} .3$ & 24 & & & see Remark 5.7(4) \\
\hline & & $Z \circ Q_{8}$ & 24 & & & TGPaley \\
\hline \multirow[t]{2}{*}{ (3) } & $G\left(7^{2}, 2\right)$ & $\operatorname{SL}(2,3)$ & 24 & 24 & $T \rtimes(Z \circ \operatorname{SL}(2,3))$ & see also \\
\hline & & $Z \circ \operatorname{SL}(2,3)$ & 72 & & & Remark 5.7(3) \\
\hline \multirow[t]{2}{*}{ (4) } & $G\left(11^{2}, 15\right)$ & $Q_{8}$ & 8 & 8 & $T \rtimes\left\langle\widehat{\omega}^{15}, \alpha\right\rangle$ & GPaley \\
\hline & & & & & & see Remark 5.7(4) \\
\hline \multirow[t]{2}{*}{ (5) } & $G\left(11^{2}, 3\right)$ & $Z \circ Q_{8}$ & 40 & 40 & $T \rtimes\left\langle\widehat{\omega}^{3}, \alpha\right\rangle$ & GPaley \\
\hline & & & & & & see Remark 5.7(4) \\
\hline \multirow[t]{2}{*}{ (6) } & $G\left(11^{2}, 5\right)$ & $\operatorname{SL}(2,3)$ & 24 & 24 & $T \rtimes \operatorname{GL}(2,3)$ & see Remark 5.7(4) \\
\hline & & $\operatorname{GL}(2,3)$ & 48 & & & \\
\hline (7) & $G\left(23^{2}, 66\right)$ & $Q_{8}$ & 8 & 8 & $T \rtimes Q_{8}$ & see Remark 5.7(4) \\
\hline (8) & $G\left(23^{2}, 6\right)$ & $Z \circ Q_{8}$ & 88 & 88 & $T \rtimes\left(Z \circ Q_{8}\right)$ & see Remark 5.7(4) \\
\hline (9) & $G\left(23^{2}, 22\right)$ & $\operatorname{SL}(2,3)$ & 24 & 24 & $T \rtimes \mathrm{SL}(2,3)$ & see Remark 5.7(4) \\
\hline (10) & $G\left(23^{2}, 11\right)$ & $\operatorname{SL}(2,3) \cdot \mathbb{Z}_{2}$ & 48 & 48 & $T \rtimes\left(\operatorname{SL}(2,3) \cdot \mathbb{Z}_{2}\right)$ & see Remark 5.7(4) \\
\hline (11) & $G\left(23^{2}, 2\right)$ & $Z \circ \mathrm{SL}(2,3)$ & 264 & 264 & $T \rtimes(Z \circ \operatorname{SL}(2,3))$ & see Remark 5.7(3) \\
\hline
\end{tabular}

that $\left(K_{49}, \mathcal{E}(M)\right)$ is also an $(H, L)$-homogeneous arc-transitive factorisation with $H=T \rtimes H_{0}, L=T \rtimes L_{0}, L_{0}<H_{0} \leq\langle\widehat{\omega}, \alpha\rangle$. Now $G(49,6)$ is not a generalised Paley graph by Proposition 4.18 , and hence by Lemmas 4.3 and $4.11, L_{0} \not \leq\langle\widehat{\omega}\rangle$ and $\alpha \notin L_{0}$; and we may assume that $(H, L)$ is admissible as in Condition 4.12. Let $H_{0}=\left\langle\widehat{\omega}^{d}, \widehat{\omega}^{e} \alpha^{s}\right\rangle$ and $L_{0}=\left\langle\widehat{\omega}^{d_{1}}, \widehat{\omega}^{e_{i}} \alpha^{s_{1}}\right\rangle$ in standard form. Since $L_{0} \not \leq\langle\widehat{\omega}\rangle$ and $\alpha \notin L_{0}$ we must have $s_{1}=s=1$ and $e_{1}>0$. Since $G(49,6)$ is $L$-arc-transitive of valency $8, d_{1} \mid 12$. Since $H_{0}$ permutes transitively the 6 factors and $L_{0} \not \leq\langle\widehat{\omega}\rangle$, it follows that $d_{1}=6 d$ with $d=1$ or 2. Suppose that $d_{1}=6$. Then by Condition 4.12(3), $3 \mid e_{1}$ and since $0<e_{1}<d_{1}$ we have $e_{1}=3$. Then the integer $c$ determined in Condition 4.12 is $c=2$ implying that $L_{0}$ has $k=d_{1} / c=3$ orbits in $V^{*}$, a contradiction. Thus $d=2, d_{1}=12$. Since $(d, e) \neq(1,0)$, by Condition 4.12(9) we have $e=1$, so $H_{0}=\left\langle\widehat{\omega}^{2}, \widehat{\omega}^{3} \alpha\right\rangle$. By Condition 4.12(3), $3 \mid e_{1}$, and by Condition 4.12(8), $2 \mid\left(1+e_{1}\right)$ so $e_{1}=3$ or 9 . The two subgroups $\left\langle\widehat{\omega}^{12}, \widehat{\omega}^{3} \alpha\right\rangle$ and $\left\langle\widehat{\omega}^{12}, \widehat{\omega}^{9} \alpha\right\rangle$ are conjugate under $\alpha$, so we may assume that $L_{0}$ is the former. A computation in MAGMA [8] verified that $A:=\operatorname{Aut}(G(49,6))=L .3$, that $A$ is contained in $Z \circ G$, and that $A$ leaves invariant the edge-partition $\operatorname{TCyc}(49,6)$. (Note that $A=T \rtimes A_{0}$ and $\bar{A}=A_{4}$.) Thus TCyc $(49,6)$ is invariant under the actions of both $A$ and the group $H$ (used to define 
TCyc $(49,6)$ in Proposition 4.18). Moreover, $\langle A, H\rangle=Z \circ G$ and we conclude that $\mathcal{E}(M)=\operatorname{TCyc}(49,6)$.

In lines (4) and (5) of Table 3, $G(121, k)$ is a generalised Paley graph with $k=15$ or 3 respectively, and we may assume that $G_{0}$ projects to $\overline{G_{0}}=A_{4}$ modulo $Z$. Suppose that $\left(K_{121}, \mathcal{E}(M)\right)$ is also an $(H, L)$-homogeneous arc-transitive factorisation with $H=T \rtimes H_{0}, L=T \rtimes L_{0}, L_{0}<H_{0} \leq\langle\widehat{\omega}, \alpha\rangle$. Since there are either 15 factors of valency 8 , or 3 factors of valency 40 , it follows that $H_{0}$ must contain $\left\langle\widehat{\omega}^{2}\right\rangle$ of order 60. Thus $\overline{H_{0}}$ contains $\mathbb{Z}_{6}$. This implies that $\left\langle G_{0}, H_{0}\right\rangle$ contains $\operatorname{PSL}(2,11)$ which cannot permute a set of 3 or 15 factors transitively.

Similarly in lines (7) or (8) of Table $3, M=\operatorname{Aut}\left(G\left(23^{2}, k\right)\right)$ is arc-regular, and isomorphic to an orbital graph of a subgroup of $\operatorname{A\Gamma L}\left(1,23^{2}\right)$. An exactly analogous argument to the one in the previous paragraph proves that the factorisation $\left(K_{23^{2}}, \mathcal{E}(M)\right)$ is not equivalent to one arising from a pair of subgroups $(H, L)$ of $\mathrm{A} \Gamma \mathrm{L}\left(1,23^{2}\right)$.

We claim that in each of the remaining cases, namely lines (2)-(4) of Table 2 or lines (6), (9)-(11) of Table 3, no subgroup of $\mathrm{A \Gamma L}\left(1, q^{2}\right)$ acts arc-transitively on the graph $G\left(q^{2}, k\right)$. This claim implies that $\left(K_{q^{2}}, \mathcal{E}(M)\right)$ does not arise in any other Part of Theorem 1.1, proving the lemma. Suppose to the contrary that $A:=$ $\operatorname{Aut}\left(G\left(q^{2}, k\right)\right)=T \rtimes A_{0}$ admits an arc-transitive action of a subgroup $H=T \rtimes H_{0}$ where $H_{0} \leq\langle\widehat{\omega}, \alpha\rangle$. We consider each of the lines in turn.

Lines (2), (3) or (4) of Table 2. Here the graphs $G\left(q^{2}, k\right)$ have valency 120 , and automorphism subgroup $A_{0}=Y \circ \operatorname{SL}(2,5)$, for a subgroup $Y \leq Z$ of order $6,4,1$ respectively. For $H$ to be arc-transitive, $H_{0}$ must contain a cyclic subgroup of order 60. However $A_{0}$ has no such subgroup.

Lines (6), (9), (10) or (11) of Table 3. Here we checked, using MAGMA, that the orders of cyclic subgroups of $A_{0}$ were at most 8 in the case of lines (6), (9) or (10), and at most 66 for line (11). On the other hand, for $H$ to be arc-transitive, $H_{0}$ must contain a cyclic subgroup of order at least $\left(q^{2}-1\right) / 2 k$ which is $12,12,24,132$ respectively. This is a contradiction.

To complete this subsection we make a series of remarks about the graphs $G\left(q^{2}, k\right)$, and the information given in Tables 2 and 3 .

\section{Remark 5.7}

(1) The graph $G\left(q^{2}, k\right)=\Gamma(\mathcal{O})$ for the non-trivial selfpaired $M$-orbital $\mathcal{O}=(\mathbf{0}, v)^{M}$ (see Definition 5.4). It has valency $\frac{q^{2}-1}{k}$, and by Lemma 5.3 is connected. Distinct pairs $(q, k)$ correspond to non-isomorphic such graphs as the orders or valencies would be different.

(2) Different groups $M_{0}$ may give rise to the same graph $G\left(q^{2}, k\right)$, as noted in Tables 2 and 3. The tables also list the automorphism groups of the $G\left(q^{2}, k\right)$ which were computed using MAGMA. In particular, in lines (7) and (8) of Table 3, we found by construction a subgroup $K \leq \mathrm{A} \Gamma \mathrm{L}\left(1,23^{2}\right)$ and a $K$-orbital graph $\Sigma \cong G\left(23^{2}, k\right)$ such that $K=\operatorname{Aut}(\Sigma)$, whence $M=\operatorname{Aut}\left(G\left(23^{2}, k\right)\right.$. (Note that by $X \cdot Y$, we mean an extension of $X$ by $Y$, while $X \circ Y$ denotes a central product.)

(3) If $k=2$, that is, in line (1) of Table 2, or line (3) or (11) of Table 3, then $G\left(q^{2}, k\right)$ is an arc-transitive self-complementary graph. These are the three exceptional 
graphs studied in [27], where they are denoted by $G\left(q^{2}\right)$, for $q=9,7,23$ respectively. Persert showed that the first two graphs are twisted generalised Paley graphs, while the third is "new" in the sense that it is neither a Paley graph nor a twisted generalised Paley graph, see [27, Lemmas 6.6-6.8] and Lemma 5.6.

(4) Isomorphisms between $G\left(q^{2}, k\right)$ and a generalised, or twisted generalised Paley graph were determined using MAGMA, and are denoted in the "Remarks" column of Tables 2 and 3 by GPaley or TGPaley respectively. Whether or not the edge-partitions are the usual cyclotomic or twisted cyclotomic partitions is determined in Lemma 5.6. Line (2) of Table 3 is exceptional in that $\mathcal{E}(M)$ is the corresponding twisted cyclotomic partition, and this is the only example with $k>2$ for which this occurs.

(5) In line (1) of Table 3, $G\left(q^{2}, k\right)$ is also isomorphic to the Hamming graph $H(5,2)$ by [25, Theorem 1.3(2)].

\subsection{Case 2(h)}

Here $\mathbf{E} \unlhd G_{0} \leq \operatorname{GL}(4,3)$, where $\mathbf{E}=2^{1+4}$ is extraspecial subgroup of order $2^{5}$. Now $G_{0} \leq N:=N_{\mathrm{GL}(4,3)}(\mathbf{E})$, and modulo the centre $Z$ we have $\overline{\mathbf{E}} \leq \overline{G_{0}} \leq \bar{N}$, where $\overline{\mathbf{E}} \cong \mathbb{Z}_{2}^{4}$. Also $N=\mathbf{E} H$ where $Z<H$ and $\bar{H} \cong S_{5}$. We find the possibilities for the groups $G_{0}$ and $M_{0}$.

Lemma $5.8 k=5, M_{0}=\mathbf{E}$ and $G_{0}=\mathbf{E} L$, where $Z<L \leq H$ and $\bar{L}=\mathbb{Z}_{5}, D_{10}$, $F_{20}, A_{5}$ or $S_{5}$.

Proof Now $G_{0}=\mathbf{E} L$ where $Z<L \leq H$. The group $\overline{\mathbf{E}}$ has 5 orbits of length 8 in the set $P_{1}(V)$ of 1 -spaces in $V=\mathbb{F}_{3}^{4}$. Since $\overline{G_{0}}$ is transitive on $P_{1}(V)$ it follows that the subgroup $\bar{L}$ is one of $\mathbb{Z}_{5}, D_{10}, F_{20}, A_{5}$ or $S_{5}$. In particular this implies that $\overline{\mathbf{E}}$ is the unique minimal normal sugroup of $\overline{G_{0}}$. Since by assumption $M_{0} \not Z Z, \overline{M_{0}}$ is a non-trivial normal subgroup of $\overline{G_{0}}$. Hence $M_{0}$ contains $\mathbf{E}$.

Now $G_{0}$ permutes the $M_{0}$-orbits in $V^{*}$ transitively, and these $M_{0}$-orbits are unions of $\mathbf{E}$-orbits. However, the group $\mathbf{E}$ has 5 orbits of length 16 in $V^{*}$, which are permuted primitively by $G_{0}$. Since $M_{0}$ is intransitive on $V^{*}$, it follows that $M_{0}$ and $\mathbf{E}$ have the same orbits in $V^{*}$, and hence that $k=5$ and $M_{0}=\mathbf{E}$.

It follows from this lemma that there is only one edge partition arising in this case, namely $\mathcal{E}(M)$ for the unique group $M=T \rtimes \mathbf{E}$. The factors $\Gamma_{i}$ of this factorisation were identified in [23] as Hamming graphs $H(9,2)$. Thus we have the following proposition, which completes the proof of Theorem 1.1 .

Proposition 5.9 Let $\left(K_{q^{2}}, \mathcal{E}\right)$ be a $(G, M)$-homogeneous arc-transitive factorisation of index $k$ such that $G=T \rtimes G_{0}$, with $G_{0}$ as in Theorem $2.5(2)(h), M_{0} \not \leq Z$ and $\phi \in$ $M_{0}$. Then $k=5, M_{0}=\mathbf{E}=2^{1+4}, \mathcal{E}=\mathcal{E}(M)$, and each $\Gamma_{i} \cong H(9,2)$. Also $G_{0}=\mathbf{E} L$, where $Z<L$ and $\bar{L}=\mathbb{Z}_{5}, D_{10}, F_{20}, A_{5}$ or $S_{5}$. In particular, Theorem $1.1(2)(\mathrm{d})$ holds. 


\section{References}

1. Alspach, B., Morris, J., Vilfred, V.: Self-complementary circulant graphs. Ars Combinatoria 53, 187191 (1999)

2. Biggs, N.: Algebraic Graph Theory. Cambridge University Press, Cambridge (1993)

3. Bonisoli, A., Buratti, M., Mazzuoccolo, G.: Doubly transitive 2-factorizations. J. Combin. Designs 15(2), 120-132 (2007)

4. Bonisoli, A., Labbate, D.: One-factorizations of complete graphs with vertex-regular automorphism groups. J. Combin. Designs 10(1), 1-16 (2002)

5. Brouwer, A.E., Cohen, A.M., Neumaier, A.: Distance Regular Graphs. Springer, Berlin (1989)

6. Cameron, P.J.: Finite permutation groups and finite simple groups. Bull. London Math. Soc. 13, 1-22 (1981)

7. Cameron, P.J.: Combinatorics: Topics, Techniques, Algorithms. Cambridge University Press, Cambridge (1994)

8. Cannon, J., Playoust, C.: An Introduction to Magma. School of Mathematics and Statistics, University of Sydney. (See also http://magma.maths.usyd.edu.au/)

9. Dixon, J.D., Mortimer, B.: Permutation Groups. Springer, New York (1996)

10. Foulser, D.A.: The flag-transitive collineation group of the finite Desarguesian affine planes. Canad. J. Math. 16, 443-472 (1964)

11. Foulser, D.A., Kallaher, M.J.: Solvable, flag-transitive, rank 3 collineation groups. Geom. Ded. 7, 111-130 (1978)

12. Giudici, M., Li, C.H., Potočnik, P., Praeger, C.E.: Homogeneous factorisations of graphs and digraphs. European J. Combin. 27, 11-37 (2006)

13. Harary, F., Robinson, R.W., Wormald, N.C.: Isomorphic factorisations I: Complete graphs. Trans. Amer. Math. Soc. 242, 243-260 (1978)

14. Harary, F., Robinson, R.W.: Isomorphic factorisations X: Unsolved problems. J. Graph Theory $\mathbf{9}$, 67-86 (1985)

15. Huppert, B.: Endliche Gruppen I. Springer, Berlin (1967)

16. Huppert, B., Blackburn, N.: Finite Groups III. Springer, Berlin (1982)

17. Jajcay, R., Li, C.H.: Constructions of self-complementary circulants with no multiplicative isomorphisms. European J. Combin. 22, 1093-1100 (2001)

18. Kantor, W.M.: Automorphism groups of designs. Math. Z. 109, 246-252 (1969)

19. Kantor, W.M.: Homogeneous designs and geometric lattices. J. Combin. Theory Ser. A 38, 66-74 (1985)

20. Li, C.H.: On self-complementary vertex-transitive graphs. Comm. Algebra 25, 3903-3908 (1997)

21. Li, C.H., Praeger, C.E.: On partitioning the orbitals of a transitive permutation group. Trans. Amer. Math. Soc. 355, 637-653 (2003)

22. Liebeck, M.W.: The affine permutation groups of rank 3. Proc. London Math. Soc. 54, 477-516 (1987)

23. Lim, T.K.: Arc-transitive homogeneous factorizations and affine planes. J. Combin. Designs 14, 290$300(2008)$

24. Lim, T.K.: Edge-transitive homogeneous factorisations of complete graphs. $\mathrm{PhD}$ thesis, University of Western Australia (2004). (http://theses.library.uwa.edu.au/adt-WU2004.0039/)

25. Lim, T.K., Praeger, C.E.: On generalised Paley graphs and their automorphism groups. Mich. Math. J. (2008, to appear)

26. Muzychuk, M.: On Sylow's subgraphs of vertex-transitive self-complementary graphs. Bull. London Math. Soc. 31, 531-533 (1999)

27. Peisert, W.: All self-complementary symmetric graphs. J. Algebra 240, 209-229 (2001)

28. Praeger, C.E.: Finite transitive permutation groups and finite vertex-transitive graphs. In: Graph Symmetry (Montrael, PQ, 1996). Kluwer Academic, Dordrecht (1997)

29. Sibley, T.Q.: On classifying finite edge colored graphs with two transitive automorphism groups. J. Combin. Theory Ser. B 90, 121-138 (2004)

30. Suprunenko, D.A.: Self-complementary graphs. Cybernetics 21, 559-567 (1985)

31. Suzuki, M.: Group Theory I. Springer, New York (1982)

32. Tutte, W.T.: Connectivity in Graphs. University of Toronto Press, Toronto (1966)

33. Wielandt, H.: Finite Permutation Groups. Academic Press, New York (1964)

34. Zhang, H.: Self-complementary symmetric graphs. J. Graph Theory 16, 1-5 (1992)

35. Zsigmondy, K.: Zur Theorie der Potenzreste. Monatsh. Math. Phys. 3, 265-284 (1892) 